\title{
New massive members of Cygnus OB2
}

\author{
S. R. Berlanas ${ }^{1,2}$, A. Herrero ${ }^{1,2}$, F. Comerón ${ }^{3}$, A. Pasquali ${ }^{4}$, C. Bertelli Motta ${ }^{4}$, and A. Sota ${ }^{5}$ \\ ${ }^{1}$ Instituto de Astrofísica de Canarias, 38200 La Laguna, Tenerife, Spain \\ e-mail: srberlan@iac.es \\ 2 Departamento de Astrofísica, Universidad de La Laguna, 38205 La Laguna, Tenerife, Spain \\ ${ }^{3}$ ESO, Karl-Schwarzschild-Strasse 2, 85748 Garching bei München, Germany \\ ${ }^{4}$ Astronomisches Rechen-Institut, Zentrum für Astronomie der Universität Heidelberg, Mönchhofstr 12-14, 69120 Heidelberg, \\ Germany \\ ${ }^{5}$ Instituto de Astrofísica de Andalucía-CSIC, 18008 Granada, Spain
}

Received 29 August 2017 / Accepted 11 November 2017

\begin{abstract}
Context. The Cygnus complex is one of the most powerful star forming regions at a close distance from the Sun $(\sim 1.4 \mathrm{kpc})$. Its richest OB association Cygnus OB2 is known to harbor many tens of O-type stars and hundreds of B-type stars, providing a large homogeneous population of OB stars that can be analyzed. Many studies of its massive population have been developed in the last decades, although the total number of OB stars is still incomplete.

Aims. Our aim is to increase the sample of $\mathrm{O}$ and B members of Cygnus OB2 and its surroundings by spectroscopically classifying 61 candidates as possible OB-type members of Cygnus OB2, using new intermediate resolution spectroscopy.

Methods. We have obtained intermediate resolution ( $~ ~ 5000)$ spectra for all of the OB-type candidates between 2013 and 2017 . We thus performed a spectral classification of the sample using HeI-II and metal lines rates, as well as the Marxist Ghost Buster (MGB) software for O-type stars and the IACOB standards catalog for B-type stars.

Results. From the whole sample of 61 candidates, we have classified 42 stars as new massive OB-type stars, earlier than B3, in Cygnus OB2 and surroundings, including 11 O-type stars. The other candidates are discarded as they display later spectral types inconsistent with membership in the association. We have also obtained visual extinctions for all the new confirmed massive OB members, placing them in a Hertzsprung-Russell Diagram using calibrations for $T_{\text {eff }}$ and luminosity. Finally, we have studied the age and extinction distribution of our sample within the region.

Conclusions. We have obtained new blue intermediate-resolution spectra suitable for spectral classification of $61 \mathrm{OB}$ candidates in Cygnus OB2 and surroundings. The confirmation of 42 new OB massive stars (earlier than B3) in the region allows us to increase the young massive population known in the field. We have also confirmed the correlation between age and Galactic longitude previously found in the region. We conclude that many $\mathrm{O}$ and early $\mathrm{B}$ stars at $B>16$ mag are still undiscovered in Cygnus.
\end{abstract}

Key words. stars: early-type - stars: massive - open clusters and associations: individual: Cygnus OB2 -

Hertzsprung-Russell and C-M diagrams - Galaxy: stellar content

\section{Introduction}

The Cygnus region is the most powerful nearby stellar complex, conspicuous at all wavelengths and very young, with several rich $\mathrm{OB}$ associations, numerous young open clusters and tens of compact HII and star formation regions in the field. Hosting the largest number of nearby massive stars and an intense star forming activity (Reipurth \& Schneider 2008), it provides an updated view of the high-mass stellar population in one of the largest groups of young stars in our Galaxy. It is an ideal place to study the process of massive star formation and evolution, individually and in stellar groups, and their interaction with the surroundings.

Its association Cygnus OB2 (d 1.4 kpc; Rygl et al. 2012) has received a lot of attention and has been studied at all wavelengths with different spatial coverage since it hosts a high number of early spectral type stars (Walborn et al. 2002). First studies were carried out by Morgan et al. (1954), Schulte (1956, 1958) and Reddish (1968), but it was Massey \& Thompson (1991) who developed an extensive survey of the massive population in the association, identifying 120 possible massive star members, 70 of which were classified as OB stars (42 O-type stars). Knödlseder (2000) proposed that this number should be much larger, around 100 O-type stars. In the last few decades, many other studies were carried out in the region updating continually this number (Comerón et al. 2002, 2008; Hanson 2003; Kiminki et al. 2007; Negueruela et al. 2008; Comerón $\&$ Pasquali 2012). These surveys have allowed the global study of the massive population in the region, using photometry to place the stars in a Hertzsprung-Russell diagram (HRD) from which the star formation history and mass function of the association were assessed (Wright et al. 2015). In spite of the many photometric and spectroscopic surveys carried out in the region, only a small homogeneous group of early type stars have been spectroscopically analyzed (Herrero et al. 1999, 2002; Negueruela et al. 2008), and few stars have been observed in the UV range (Herrero et al. 2001). There is still a large number of stars that should be explored. The optical extinction of the region is high $\left(A_{\mathrm{V}}=4.0-7.0 \mathrm{mag}\right.$; Wright et al. 2015), but not so much as to prevent obtaining spectra of its most massive stars for a rough spectral classification. Therefore, new spectroscopy to search for previously undiscovered massive stars is mandatory to complete the last census of massive $\mathrm{O}$ and B-type stars in the association. 
Table 1. Telescopes, instruments and settings used in this work.

\begin{tabular}{lcccc}
\hline \hline $\begin{array}{l}\text { Instrument } \\
\text { and grating }\end{array}$ & Telescope & Resol. & Date & Stars \\
\hline ISIS - 600B & ORM-WHT & 2500 & Oct. 2013 & 1 \\
WYFFOS - H2400B & ORM-WHT & 5000 & Jul. 2014 & 7 \\
ISIS - H2400 & ORM-WHT & 7500 & Jul. 2015 & 2 \\
OSIRIS - R2500U/V & ORM-GTC & 2500 & May 2016 & 2 \\
IDS - R1200B & ORM-INT & 5000 & Jul. 2016 & 47 \\
ISIS - R1200B & ORM-WHT & 5000 & Apr. 2017 & 2 \\
\hline
\end{tabular}

One of the most complete spectroscopic surveys in the Cygnus region was developed by Comerón \& Pasquali (2012). They performed spectral classification of a magnitude-limited sample ( $B \leq 16 \mathrm{mag}$ and $K s<9$ mag) selected with a homogeneous photometric criterion over a large area that includes Cygnus OB2 and its surroundings, providing a large sample of known and new OB stars, as well as a list of $61 \mathrm{OB}$ candidates for which no spectral data is available and that are pending spectroscopic confirmation.

The main goal of this work is to complete the spectral classification of this latter sample, aiming a later determination of the stellar parameters. Thus, we have obtained intermediateresolution spectra of all the list candidates, in order to confirm or reject them as true massive OB-type stars. In Sect. 2 we present the observations. In Sect. 3 we describe the spectral classification criteria used in this work, and in Sect. 4 visual extinctions and stellar parameters derived for the new OB-type members. The results are discussed in Sect. 5 where we show the HRD of the region and the age distribution found across Galactic longitude. Finally, we summarize our conclusions in Sect. 6.

\section{Observations and data reduction}

The study developed by Comerón \& Pasquali (2012) produced a sample of $\mathrm{O}$ and early $\mathrm{B}$ stars (in a $6^{\circ} \times 4^{\circ}$ region centered on Galactic coordinates $l=79.8^{\circ}$ and $b=+0.8^{\circ}$ of Cygnus OB2) which were identified using two homogeneous reddening-free criteria

$$
\begin{aligned}
& Q_{B J K}=0.196(B-J)-0.981(J-K)-0.098>0 \\
& Q_{J H K}=0.447(J-H)-0.894(H-K)-0.089<0
\end{aligned}
$$

which allowed them to classify 60 new OB stars and produce a list of 61 candidates pending spectroscopic data. They used $B J H K$ photometry tabulated in the USNO-B (Zacharias et al. 2010, 2004) and 2MASS all-sky (Skrutskie et al. 2006) catalogs setting limiting magnitudes of $B \leq 16 \mathrm{mag}$ and $K s<9.0 \mathrm{mag}$. By combining these two magnitude cuts with the $(B-K)$ colors of OB stars, Comerón \& Pasquali (2012) set up a selection method sensitive to main-sequence stars earlier than B1 and obscured by $A_{\mathrm{V}}<6.7 \mathrm{mag}$.

We have obtained new spectra for all the proposed OB candidates, whose location is shown in Fig. 1. The sample has been observed in five different runs between 2013 and 2017. For an accurate spectral classification of OB-type stars we need blue spectra (4000-5000 $\AA$ ) where the diagnostic He I-II and metal lines are located.

The bulk of stars were observed in July 2016, obtaining spectra of 47 candidates. We have chosen the R1200B grating of IDS (EEV10) on the Isaac Newton Telescope (INT) in

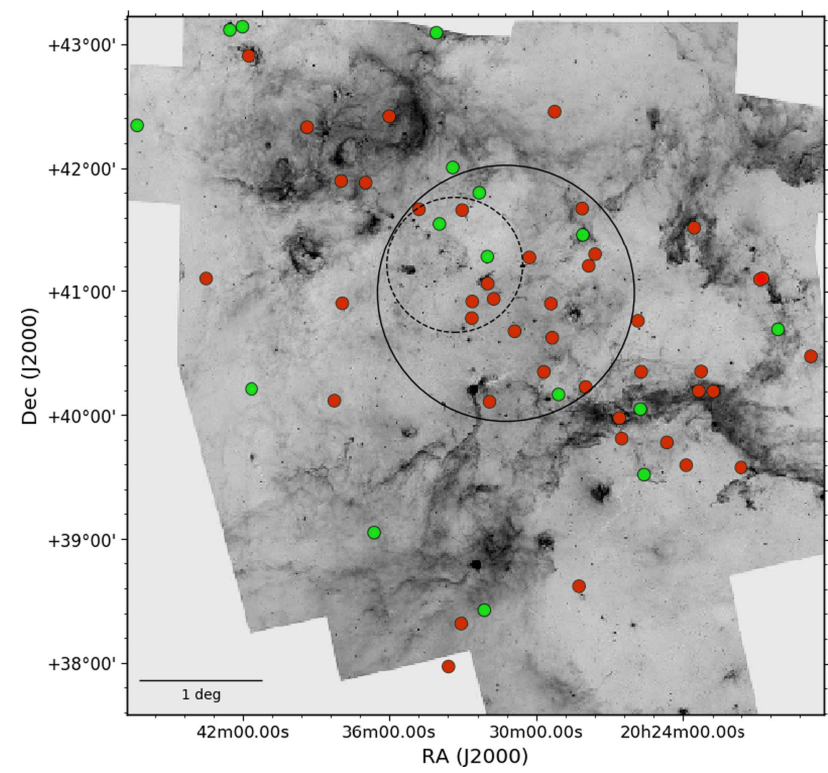

Fig. 1. Inverse Spitzer $8 \mu \mathrm{m}$ image of the Cygnus region showing the location of the $61 \mathrm{OB}$ candidates. The 42 confirmed massive OB-type stars earlier than B3 are indicated with red dots. The remaining stars are late B and foreground A-F-G stars which are indicated with green dots. The solid line circle delimits the 1 deg radius area of Cygnus OB2 adopted by Comerón \& Pasquali (2012). For reference, the dash-dotted line circle shows the area considered by Wright et al. (2015).

La Palma, which provides a resolution of $\sim 5000$ at $4500 \AA$. The remaining stars were observed in different runs between 2013 and 2017, at the William Herschel Telescope (WHT) using the AF2/WYFFOS and ISIS instruments, and at the Gran Telescopio CANARIAS (GTC) using the OSIRIS instrument. Out of the whole sample, three of the stars (J20301097+4120088, J20315433+4010067, J20345785+4143543) belong to the Galactic O-Star Spectroscopic Survey (GOSSS) catalog (Maíz Apellániz et al. 2016).

The information on the different runs carried out in this work is shown in Table 1. The final spectra were reduced using the IRAF procedures, with standard routines for bias and flat-field subtraction and also for the wavelength calibration.

\section{Spectral classification}

The accuracy of the spectral classification depends on the effects of spectral resolution as well as the signal-to-noise ratio $(\mathrm{S} / \mathrm{N})$. The main diagnostic method for O-type stars is the comparison of He II 4542/He I 4471 ratio (Sota et al. 2011). These lines are similar for a $\mathrm{O} 7$ type star. For later $\mathrm{O}$ types the relative strengths of He II 4542/He I 4387 and He II 4200/He I 4144 are normally used, and represent the main criteria for types O8-B0. Spectral types earlier than $\mathrm{O} 8$ were classified using the criteria described by Gray \& Corbally (2009). The presence of metal lines in different ionization stage, such as Si III or $\mathrm{Mg}$ II, indicates early B-type stars. The relative strength of HeII 4471/MgII 4481 is a useful indicator for B1-late B stars. As secondary indicators we used the criteria described by Gray \& Corbally (2009), which were also used to classify stars of spectral types A, F and G.

Regarding the luminosity class, the criteria used for early O-type stars were introduced by Walborn (1971, 1973), taking into account the emission effects in the He II 4686 line and N III 4634-4640-4642. For late O-type stars, we have used the criteria described by Sota et al. (2011) and for B-type stars the 
Table 2. Basic data of the confirmed massive new members (earlier than B3).

\begin{tabular}{|c|c|c|c|c|c|c|c|c|}
\hline Object & RA (hhmmss) & $\operatorname{Dec}\left({ }^{\circ}{ }^{\prime \prime \prime}\right)$ & Region & $B$ & $K s$ & $J$ & $\mathrm{SpT}$ & Binary star \\
\hline $\mathrm{J} 20423509+4256364$ & 204235.08 & +425636.43 & $c$ & 14.480 & 8.304 & 9.211 & O6IIIz & \\
\hline J20371773+4156316 & 203717.73 & +415631.57 & $c$ & 15.760 & 8.041 & 9.071 & O7V & \\
\hline J20345785+4143543 & 203457.84 & +414354.25 & $a$ & 15.430 & 7.417 & 8.447 & $\mathrm{O} 7: \mathrm{Ib}$ & \\
\hline J20293563+4024315 & 202935.63 & +402431.45 & $b$ & 12.468 & 8.268 & 8.831 & O8IIIz & \\
\hline J20222481+4013426 & 202224.81 & +401342.55 & $c$ & 13.620 & 8.410 & 9.034 & O8II & \\
\hline J20261976+3951425 & 202619.75 & +395142.46 & $c$ & 15.600 & 8.348 & 9.351 & O8.5IV & \\
\hline J20275292+4144067 & 202752.92 & +414406.65 & $b$ & 13.330 & 7.277 & 8.144 & O9.5II & \\
\hline J20262484+4001413 & 202624.84 & +400141.25 & $c$ & 13.290 & 8.330 & 9.021 & O9.2III & \\
\hline $\mathrm{J} 20291617+4057372$ & 202916.17 & +4057 37.19 & $b$ & 15.030 & 7.899 & 8.855 & O9.7III & \\
\hline $\mathrm{J} 20382173+4157069$ & 203821.72 & +415706.89 & $c$ & 15.810 & 7.682 & 8.760 & O9.7II & \\
\hline $\mathrm{J} 20181090+4029063$ & 201810.89 & +402906.29 & $c$ & 14.940 & 8.399 & 9.343 & O9.7Ib & yes* \\
\hline J20273787+4115468 & 202737.87 & +411546.79 & $b$ & 14.570 & 8.263 & 9.146 & B0II & \\
\hline $\mathrm{J} 20301097+4120088$ & 203010.97 & +412008.82 & $b$ & 15.690 & 8.882 & 9.855 & B0:II: & \\
\hline $\mathrm{J} 20323968+4050418$ & 203239.68 & +405041.83 & $a$ & 14.410 & 8.913 & 9.631 & B0II & \\
\hline $\mathrm{J} 20395358+4222506$ & 203953.58 & +422250.62 & $c$ & 15.890 & 5.822 & 7.345 & B0I & yes* \\
\hline J20281176+3840227 & 202811.75 & +384022.73 & $c$ & 11.805 & 7.944 & 8.349 & BOIb & \\
\hline J20323882+4058469 & 203238.82 & +405846.85 & $a$ & 15.430 & 8.821 & 9.701 & BOIb & \\
\hline $\mathrm{J} 20225451+4023314$ & 202254.50 & +402331.39 & $c$ & 13.107 & 8.601 & 9.175 & B0Iab & \\
\hline $\mathrm{J} 20253320+4048444$ & 202533.19 & +404844.38 & $c$ & 13.112 & 7.648 & 8.340 & B0Iab & \\
\hline $\mathrm{J} 20272099+4121262$ & 202720.99 & +412126.15 & $b$ & 13.830 & 8.730 & 9.448 & B $0.5 \mathrm{~V}$ & yes \\
\hline HDE229258 & 202425.51 & +394928.30 & $c$ & 10.235 & 8.689 & 8.833 & B $0.7 \mathrm{~V}$ & \\
\hline J20330526+4143367 & 203305.26 & +414336.74 & $a$ & 13.940 & 8.634 & 9.286 & B0.5III & \\
\hline $\mathrm{J} 20361806+4228483$ & 203618.06 & +422848.30 & $c$ & 15.650 & 8.855 & 9.814 & B0.7III & \\
\hline J20233816+3938118 & 202338.16 & +393811.84 & $c$ & 11.293 & 8.731 & 8.996 & B0.7Ib & \\
\hline HD 228973 & 202007.35 & +410746.72 & $c$ & 10.34 & 7.684 & 7.922 & $\mathrm{~B} 1 \mathrm{~V}$ & yes \\
\hline J20201435+4107155 & 202014.34 & +4107 15.45 & $c$ & 12.412 & 8.240 & 8.627 & B1V & \\
\hline J20230290+4133466 & 202302.90 & +413346.59 & $c$ & 14.780 & 7.809 & 8.762 & B1V & \\
\hline $\mathrm{BD}+404193$ & 202913.55 & +404103.38 & $b$ & 10.412 & 8.812 & 8.945 & B1V & \\
\hline $\mathrm{BD}+404208$ & 203049.97 & +404418.53 & $b$ & 10.654 & 8.664 & 8.869 & $\mathrm{~B} 1 \mathrm{~V}$ & \\
\hline $\mathrm{J} 20314341+4100021$ & 203143.40 & +410002.07 & $a$ & 15.940 & 8.957 & 9.885 & B1V & yes* \\
\hline J20315898+4107314 & 203158.98 & +410731.41 & $a$ & 15.490 & 8.832 & 9.773 & B1V & yes* \\
\hline $\mathrm{J} 20330453+3822269$ & 203304.53 & +3822 26.91 & $c$ & 11.383 & 8.790 & 9.021 & B1V & \\
\hline J20230183+4014029 & 202301.83 & +401402.90 & $c$ & 13.465 & 8.060 & 8.579 & B1III & \\
\hline J20274925+4017004 & 202749.25 & +401700.42 & $b$ & 13.460 & 8.104 & 8.713 & B1III & \\
\hline $\mathrm{J} 20315433+4010067$ & 203154.33 & +401006.71 & $b$ & 15.990 & 8.884 & 9.742 & B1III & \\
\hline $\mathrm{J} 20382889+4009566$ & 203828.88 & +400956.63 & $c$ & 9.996 & 8.816 & 8.896 & B1III & \\
\hline J20440752+4107342 & 204407.51 & +410734.18 & $c$ & 15.470 & 8.703 & 9.562 & B1III & \\
\hline LSII +3797 & 203335.52 & +380136.73 & $c$ & 11.838 & 5.900 & 6.691 & B1Ia & yes* \\
\hline J20211924+3936230 & 202119.24 & +393622.98 & $c$ & 12.732 & 7.886 & 8.488 & $\mathrm{~B} 1 \mathrm{Ib}$ & yes* \\
\hline $\mathrm{BD}+394179$ & 202527.28 & +402400.15 & $c$ & 11.066 & 6.667 & 7.210 & $\mathrm{~B} 1 \mathrm{Ib}$ & \\
\hline J20290247+4231159 & 202902.46 & +423115.91 & $c$ & 13.330 & 8.426 & 9.090 & $\mathrm{~B} 1 \mathrm{Ib}$ & yes* \\
\hline J20381289+4057169 & 203812.88 & +405716.86 & $c$ & 12.870 & 8.675 & 9.183 & $\mathrm{~B} 2 \mathrm{~V}$ & \\
\hline
\end{tabular}

Notes. $B$ magnitudes from the USNO-B catalog. $K s$ and $J$ magnitudes from 2 MASS catalog. Region $a$ indicates the 1 deg. circular area centered on Cyg OB2\#8 trapezium adopted by Wright et al. (2015) for the Cygnus OB2 association. Region $b$ indicates the 1 deg. radius area adopted by Comerón \& Pasquali (2012) for the same Cygnus OB2 association. Region $c$ indicates the surrounding area outside the 1 deg. radius adopted by Comerón \& Pasquali (2012). For binary stars asterisks indicate possible SB2 stars, whose spectral types are refered to the primary component.

criteria described by Gray \& Corbally (2009) and the Balmer lines width.

Although the classification for O-type stars was based on the described $\mathrm{He}$ and metal lines diagnostic criteria, we have also used for O-type stars the Marxist Ghost Buster (MGB) code developed by Maíz Apellániz et al. (2012) in order to obtain a more accurate result. This tool compares the observed spectra with a grid of $\mathrm{O}$ standards (in this work the GOSSS library), allowing us to vary spectral type, luminosity class, velocity and resolution until obtaining a best match. Furthermore, for B-type stars we have used a sample of IACOB standards (Simón-Díaz et al. 2015) to improve their classification.

\section{New confirmed OB type stars}

The observed spectra have high enough $\mathrm{S} / \mathrm{N}$ and resolution for a spectral classification of the stars. All the candidate spectra are plotted in Fig. B.1 where the main diagnostic lines used are also indicated. 
Table 3. Candidates classified as late B and A-F-G type stars.

\begin{tabular}{|c|c|c|}
\hline Object & RA (hhmmss) & $\operatorname{Dec}\left({ }^{\circ}{ }^{\prime \prime \prime}\right)$ \\
\hline \multicolumn{3}{|l|}{ late B-type stars } \\
\hline CCDMJ20323+4152AB & 203220.81 & +415200.78 \\
\hline $\mathrm{BD}+423785 \mathrm{a}$ & 203415.39 & +430935.28 \\
\hline \multicolumn{3}{|l|}{ A-type stars } \\
\hline $\mathrm{J} 20252497+3934030$ & 202524.96 & +393403.02 \\
\hline $\mathrm{J} 20285874+4013302$ & 202858.74 & +401330.22 \\
\hline $\mathrm{J} 20315984+4120354$ & 203159.84 & +41 2035.41 \\
\hline $\mathrm{J} 20320734+3828586$ & 203207.34 & +382858.62 \\
\hline $\mathrm{BD}+413801$ & 203330.39 & +420417.35 \\
\hline J20364336+3906145 & 203643.36 & +3906 14.53 \\
\hline CCDM J20429+4311AB & 204254.24 & +431038.71 \\
\hline \multicolumn{3}{|l|}{ F-type stars } \\
\hline $\mathrm{J} 20193232+4042447$ & 201932.32 & +404244.72 \\
\hline $\mathrm{J} 20253116+4005508$ & 202531.16 & +400550.82 \\
\hline J20275204+4131200 & 202752.03 & +4131 19.98 \\
\hline CCDM J20420+4015 & 204201.18 & +401442.70 \\
\hline $\mathrm{J} 20500396+4300118$ & 205003.96 & +430011.76 \\
\hline $\mathrm{J} 20504551+421012.6$ & 205045.51 & +421012.64 \\
\hline \multicolumn{3}{|l|}{ G-type stars } \\
\hline $\mathrm{J} 20300022+4337553$ & 203000.21 & +433755.29 \\
\hline $\mathrm{J} 20340430+4136507$ & 203404.29 & +413650.67 \\
\hline $\mathrm{J} 20432737+4308525$ & 204327.37 & +430852.47 \\
\hline $\mathrm{J} 20472235+4220523$ & 204722.34 & +422052.30 \\
\hline
\end{tabular}

Out of the 61 candidates 42 are OB type stars, earlier than B3, including 11 O-type stars. Two more are late B-type stars. The location of these new confirmed OB stars is shown in Fig. 1 while their names, coordinates, magnitudes and the derived spectral classification are listed in Table 2 . We have also included the region in which they are located: (a) the Cygnus OB2 area considered by Wright et al. (2015) which is the youngest core of Cyg OB2 at present; (b) the Cygnus OB2 area considered by Comerón \& Pasquali (2012) which is the extended Cyg OB2 area containing older stars, on average, and (c) the surrounding area which includes part of the Cygnus OB9 association and field population. The remaining observed stars are late B and foreground A-F-G stars, which are globally listed in Table 3. The selection criteria success rate obtained by Comerón \& Pasquali (2012) along with the number of the confirmed OB type obtained in this work (72\%), support the success at identifying reddened massive OB stars with this method.

We have also detected nine possible or confirmed SB2 binaries in the sample of new OB stars (see Table 2). In some cases, indicated with asterisks, we can only suggest possible binary nature mainly due to noisy spectra. This number represents a $21 \%$ of our massive OB sample. We assign to these stars the spectral classification of their primary component. An example is shown in Fig. 2. Sana \& Evans (2011) found that at least $45-55 \%$ of the $\mathrm{O}$ star population in clusters and $\mathrm{OB}$ associations is comprized of spectroscopic binaries, which indicates that it is highly likely that more binaries are undetected in our sample.

\subsection{Extinction}

We used the $(J-K s)$ colors and the recent extinction law derived by Wright et al. (2015) $\left(R_{\mathrm{V}}=2.91\right)$ to obtain individual extinctions for all the new confirmed massive OB type stars
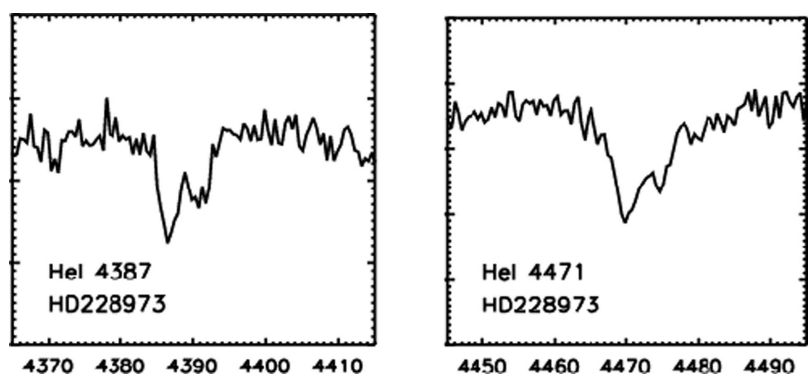

Fig. 2. Example of SB2 detection: HeI lines in star HD 228973.

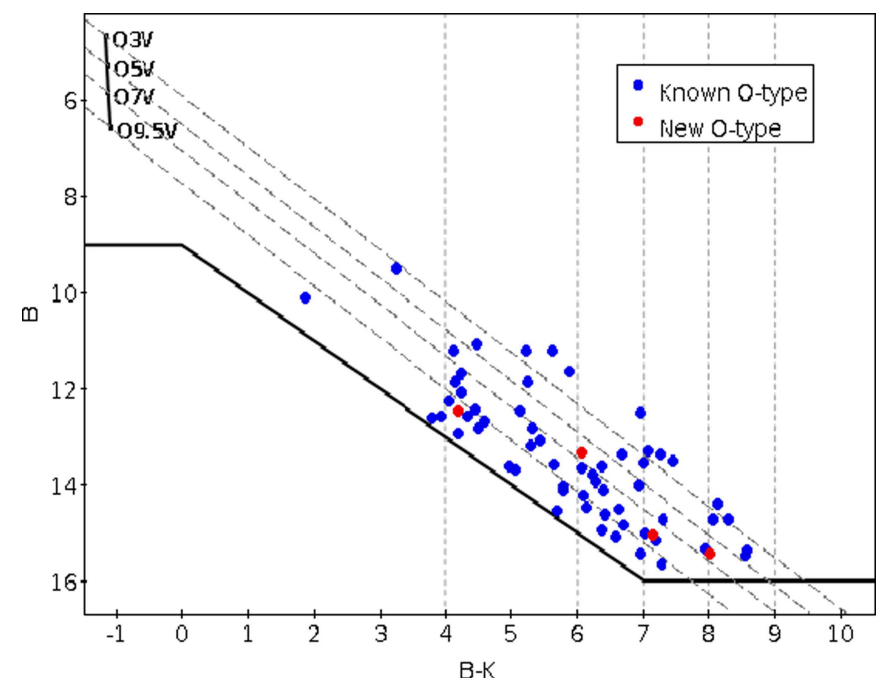

Fig. 3. $(B-K), B$ diagram of the confirmed O-type stars in Cygnus OB2 within the $1 \mathrm{deg}$. radius area adopted by Comerón \& Pasquali (2012). Blue dots indicate stars previously known, and red dots represent the new ones confirmed in this work. The upper left vertical line shows the position of the unreddened main sequence based on intrinsic magnitudes from Martins \& Plez (2006) at a distance modulus $D M=10.8 \mathrm{mag}$. The dash-dotted lines represent the locus expected for different spectral type stars using the reddening law of Wright et al. (2015) with $R_{\mathrm{V}}=2.91$. The solid line marks the limits imposed by the selection criteria at magnitudes $B \leq 16 \mathrm{mag}, K s<9$ mag. Vertical dotted lines indicate $(B-K)$ color bins.

(see Table A.1). Most of them have visual extinctions in the range $A_{\mathrm{V}}=4-8 \mathrm{mag}$, which agrees with previous studies in the region (Comerón \& Pasquali 2012; Wright et al. 2015). But, as in Comerón \& Pasquali (2012), this is partly consequence of the imposed $B \leq 16$ and $K s<9$ magnitude limits.

In Fig. 3 we present the $B,(B-K)$ diagram of the confirmed O-type stars in Cygnus OB2. We observe that some of them are aligned below the $09.5 \mathrm{~V}$ reddening vector, which could suggest a small shift in the adopted intrinsic color calibration since most of them are already known dwarf late O-type members. In spite of this we can assess the incompleteness of the $\mathrm{O}$ population in the region by taking into account the ratios of stars in different spectral (O3-O5, O5-O7 and O7-O9.5) and $(B-K)$ color bins.

All the stars of our sample with $(B-K)$ colors $<6$ mag should be bright enough to have been detected by our selection criteria. Figure 3 shows that the population is expected to be complete for $(B-K)<7$ mag. But for $(B-K)>7$ mag we can not see late O-type stars because they are fainter than $B=16 \mathrm{mag}$, which is the imposed magnitude limit. Assuming that the ratios of spectral types are independent of extinction, we can conclude we are loosing the fainter or more obscured O-type stars. 

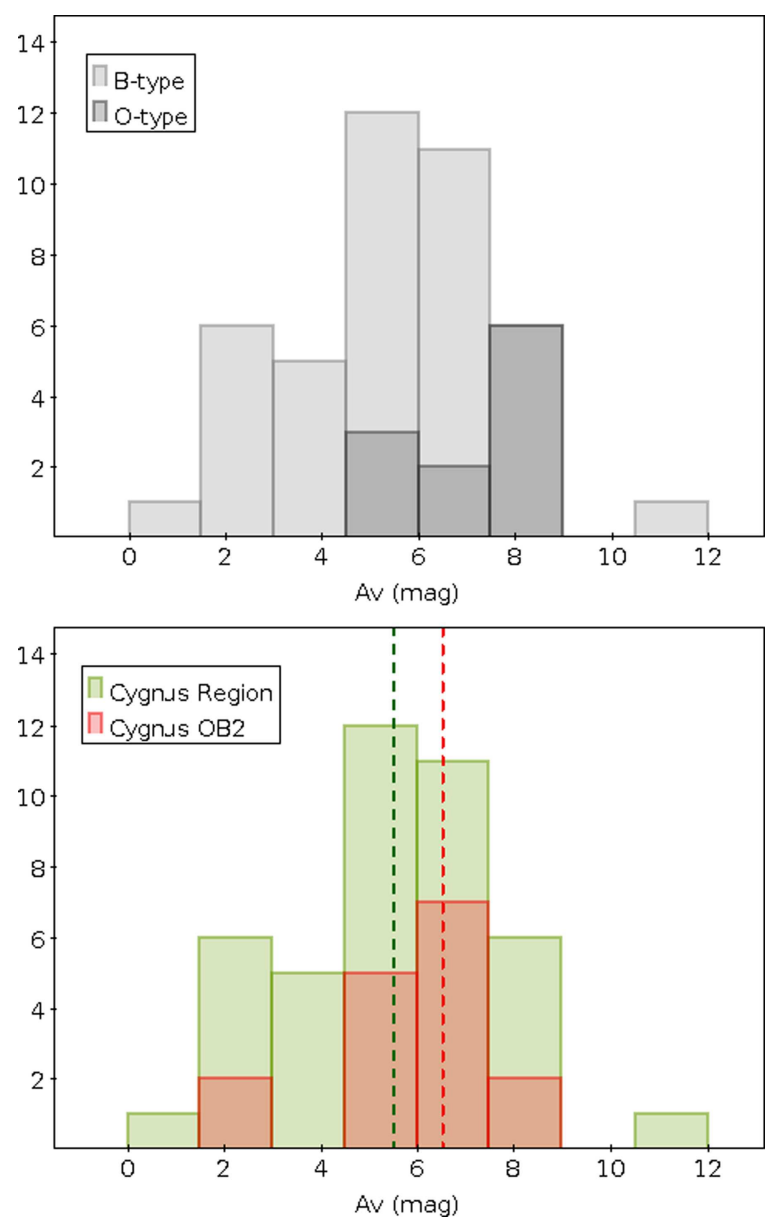

Fig. 4. Top: extinction distribution of the new classified OB type stars earlier than B3 in Cygnus OB2 and boundaries. Gray indicates B-type stars while dark gray are O-type stars. Bottom: green indicates all the confirmed stars in the Cygnus region, which includes Cyg OB2, Cyg OB9 and field. Red indicates only those new OB stars located in Cygnus OB2. Green and red dashed lines indicate the median values for the stars in the whole Cygnus region and only Cygnus OB2 respectively.

The extinction distribution for the 42 new confirmed $O B$ stars earlier than B3 in Cygnus OB2 and boundaries is shown in Fig. 4 (top). Again, the spectral classification for those stars classified as possible or confirmed SB2 binaries is taken from the primary component. Although O-type stars are clearly more obscured, a median value of $A_{\mathrm{V}}=5.5 \mathrm{mag}$ was found for the whole sample. However, this value is also affected by completeness. O-type stars are more obscured on average because we can detect them up to higher foreground extinctions thanks to their intrinsic brightness. In the bottom histogram the same sample is differentiated by location: the whole sample that belongs to Cygnus OB2, Cygnus OB9 and boundaries, and as a sub-sample, those stars located within the Cygnus OB2 area (from Comerón $\&$ Pasquali 2012) for which a median value of $A_{\mathrm{V}}=6.5 \mathrm{mag}$ is derived.

We see an abrupt cut for $A_{\mathrm{V}} \geq 9$ mag. Stars with such high visual extinctions shall be intrinsically extremely bright to be seen. An O9V star with a visual extinction of $A_{\mathrm{V}}=9 \mathrm{mag}$ will have an apparent magnitude $B \simeq 17 \mathrm{mag}$, beyond the selection criteria limits and probably this is the reason why we do not find stars beyond this value. However, for the star J20395358+4222506 classified as B0I, we have obtained a visual extinction of $11.0 \mathrm{mag}$. It has a magnitude in the $B$ band of 15.89 mag but in the $K s$ band of only $5.82 \mathrm{mag}$ which indicates

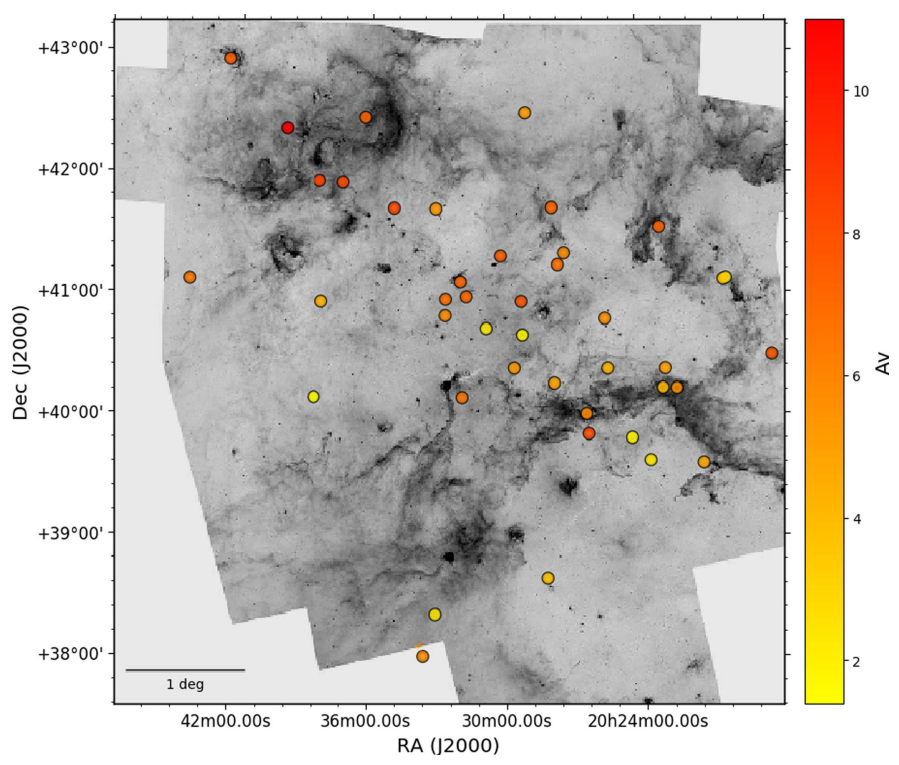

Fig. 5. Location of the new confirmed OB stars in the region colored according to the derived extinction $\left(A_{\mathrm{V}}\right)$ and over-plotted on an inverse Spitzer $8 \mu \mathrm{m}$ image.

a very bright star. These cases show again that the sample does not provide a complete census. The magnitude-limited sample of Cygnus OB2 is made incomplete due to extinction.

In Fig. 5 we present the spatial location of the new classified OB stars, where each star is color-coded according to its derived visual extinction $A_{\mathrm{V}}$. The extinction distribution varies smoothly across the region, increasing from the south-west (Cygnus OB9) to the northeast (Cygnus X-North) where the most extinguished star of our sample, J20395358+4222506, is located.

\subsection{Stellar parameters}

\subsubsection{Effective temperature}

For each classified O-type star and luminosity classes V, III and I, we have used the effective temperature $\left(T_{\text {eff }}\right)$ scale developed by Holgado et al. (in prep.) based on a revision of the O-type standards in the IACOB database. We have preferred it over the SpT- $T_{\text {eff }}$ calibration suggested by Martins et al. (2005) since the latter gives too low $T_{\text {eff }}$ values for late O-type stars (Simón-Díaz et al. 2014).

For early B-type stars and luminosity class V, we decided to use the $T_{\text {eff }}$ spectral type compilation of Nieva (2013). An excellent agreement with the calibration of Holgado et al. is found. For late B-type dwarf stars we have used the calibration of Pecaut \& Mamajek (2013). Regarding B stars with luminosity class I, we have used the $T_{\text {eff }}$ scale of Markova \& Puls (2008), and for luminosity class III we have interpolated between classes I and V. In Fig. 6 are represented the different $T_{\text {eff }}$ scales adopted in this work, and the derived $T_{\text {eff }}$ values for all the new OB type stars are shown in Table A.1. We see that all scales for B-types fit smoothly the Holgado et al. scale. Temperatures were also obtained for those stars classified as SB2 binaries by taking into account the primary component.

Comerón \& Pasquali (2012) used the "observational" effective temperature based on the $T_{\text {eff }}$ versus spectral type calibration of Martins et al. (2005). For B-type stars, they used the $T_{\text {eff }}$ spectral type compilation of Tokunaga (2000) but applying a scaling factor to force the agreement with Martins et al. (2005) temperature scale. In order to be consistent with them we have 


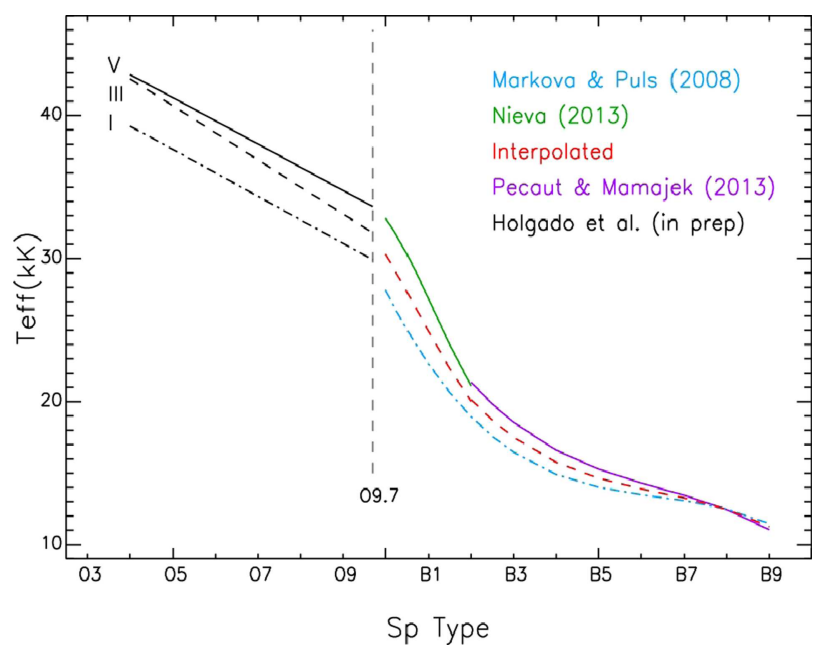

Fig. 6. $T_{\text {eff }}$ scales used in this work.

recalculated temperatures for all of their known OB stars sample in the Cygnus Region using our criteria. New values are shown in Table A.2.

\subsubsection{Bolometric correction and luminosity}

As Comerón \& Pasquali (2012), we have adopted a distance modulus of $D M=10.8 \mathrm{mag}$, and used the intrinsic magnitudes derived by Martins \& Plez (2006) for O stars and those compiled by Tokunaga (2000) for B stars. Thus we could derive absolute magnitudes (see Comerón et al. 2008). To derive luminosities we adopted bolometric corrections (BCs) from Lanz \& Hubeny (2003, 2007). There is an excellent agreement with Martins et al. (2005) for temperatures higher than $\sim 32000 \mathrm{~K}$, and also with the bolometric corrections from Nieva (2013) for cooler stars (see Nieva 2013). The derived luminosity values for the new and already known OB stars are shown in Table A.1 and Table A.2, respectively.

\section{Discussion}

Some of the new confirmed OB type stars (see Fig. 1) are located at the boundaries assigned to Cygnus OB2. However, the limits of the association are not strongly defined. The first surveys in the association assumed a smaller area where the most luminous members are located (Münch \& Morgan 1953; Massey \& Thompson 1991). Then, Knödlseder (2000) provided wider limits showing that extinction was a limiting factor in previous studies. This area was thus extended with the identification of new early-type members (Comerón et al. 2002, 2008; Comerón \& Pasquali 2012; Wright et al. 2009; Wright et al. 2015). If we assume the extension of Cygnus OB2 as the area adopted by Comerón \& Pasquali (2012) (1 deg. radius centered on Galactic coordinates $l=79.8^{\circ}$ and $b=+0.8^{\circ}$ ), as well as the most recent census in the area developed by Wright et al. (2015), we can now update the census of $\mathrm{O}$ and B-type stars in Cygnus OB2 from 204 to 221, and the number of confirmed O-type members increases from 66 to 70 stars.

\subsection{Hertzsprung-Russell diagram}

Some studies about the age of Cygnus OB2 have been developed in the last two decades (Massey et al. 2001; Hanson 2003; Drew et al. 2008; Negueruela et al. 2008; Wright et al. 2010;
Comerón \& Pasquali 2012; Comerón et al. 2016). However, different evolutionary stellar models were used. We decided to explore four different stellar models (two families with two initial rotational velocities) to construct the HRD in order to assess uncertainties on the age distribution.

We have placed the new confirmed OB stars in the HRD to study the evolutionary status of the association. For this aim we have considered the stars within the area adopted by Comerón \& Pasquali (2012). We have also added the already known OB stars compiled in their work to complete the sample. Two of them, classified as B0 stars (J20325964+4115146 and J20333700+4116113) were recently reclassified as O9.7III and O9.5IV by Maíz Apellániz et al. (2016). We thus decided to assume this last classification for both. We have used the Geneva evolutionary tracks and isochrones with and without rotation as calculated by Ekström et al. (2012) (Fig. 7, top), and for comparison, the Bonn evolutionary tracks and isochrones with and without rotation as calculated by Brott et al. (2011) (Fig. 7, bottom).

In the Geneva case, we found a difference up to $\sim 2 \mathrm{Myr}$ in the stellar ages for those most massive members $\left(\geq 20 M_{\odot}\right)$ depending on whether we consider rotation or not. Larger stellar lifetimes are derived from rotating stellar models. A similar result was found by Wright et al. (2015) by comparing both nonrotating and rotating Geneva stellar models. They derived an age range of 1-7 Myr for the association, but the results from rotating stellar models suggested an age of 4-5 Myr, while non-rotating models hinted at a younger age ( $\sim 2-3 \mathrm{Myr})$. On the contrary, the Bonn stellar models included on this work do not exhibit a large difference in the estimated age of the stars when they include or not stellar rotation. This different behavior is most likely to be ascribed to their different treatment of rotation. Thus, the positions of the isochrones in both Bonn HR diagrams are very similar. Moreover, in the Bonn case we see an extended terminal age main sequence (TAMS), and therefore, all the OB stars are located on the main sequence (MS). In spite of this, all models suggest that most of the stars are in the age range of 1-6 Myr, indicating a continuous (but not necessarily constant) star formation activity. However, a combination of different scenarios (rotating and non-rotating stellar models) would help to narrow the possible ages. Slow rotators with initial masses of $30 M_{\odot}$ or less would give similar ages as more massive stars born with larger rotational velocities.

In view of the results, we can conclude that uncertainties due to rotation and adopted models affect mainly the most massive members, suggesting larger ages of about 1-2 Myr for them. This makes the age determination very uncertain and strengthens the need for additional diagnostics, in particular individual rotation velocity measurements, to better constrain them.

\subsection{Age distribution across Galactic longitude}

Comerón \& Pasquali (2012) studied the correlation between ages and Galactic longitudes in Cygnus OB2. They suggest that star formation has proceeded from lower to higher Galactic longitudes, finding most of the old stars located at low Galactic longitudes while the youngest ones lie at higher Galactic longitudes. In order to corroborate it, we have obtained ages by comparing isochrones from the HRDs, and divided the sample of OB stars in three age groups: the young group contains stars in the range of 0-5 Myr, the intermediate group contains stars in the range of 5-10 Myr, and the old one contains stars with ages $>10$ Myr. 

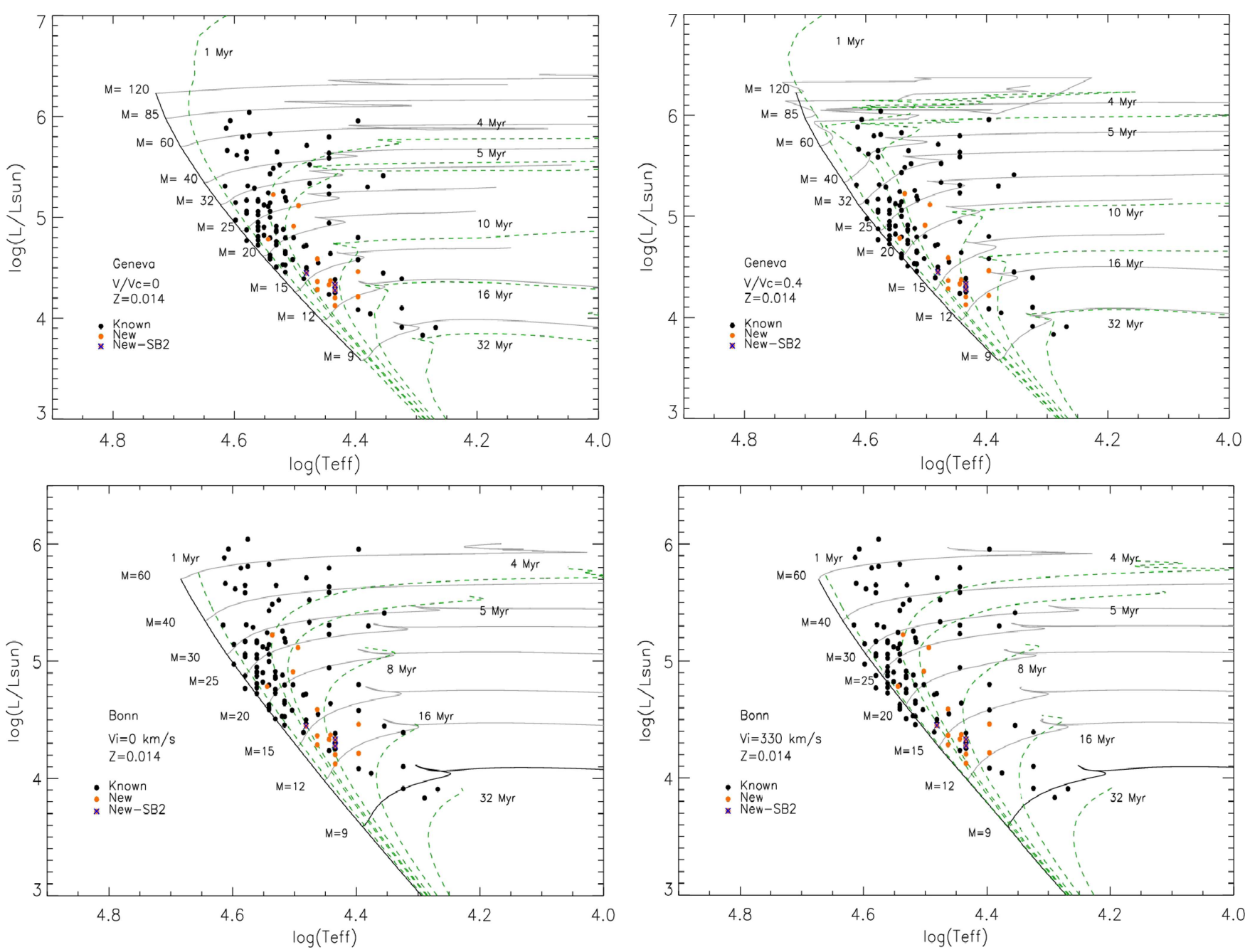

Fig. 7. HR diagrams of OB stars in Cygnus OB2 assuming a DM = 10.8 mag. Known OB stars from Comerón \& Pasquali (2012) are also included to complete the sample. Black and orange dots indicate the already known and new OB-type stars respectively. Blue crosses indicate those new stars classified as possible SB2 stars. Top left hand panel: isochrones (dotted lines) and evolutionary stellar tracks (solid lines) for non-rotating models from Ekström et al. (2012). Top right hand panel: isochrones (dotted lines) and evolutionary stellar tracks (solid lines) for rotating models (V/ $\mathrm{V}_{\mathrm{c}}$ $=0.4$ ) from Ekström et al. (2012) . Bottom left hand panel: isochrones (dotted lines) and evolutionary stellar tracks (solid lines) for non-rotating models from Brott et al. (2011). Bottom right hand panel: isochrones (dotted lines) and evolutionary stellar tracks (solid lines) for rotating models $\left(\mathrm{V}_{\mathrm{i}}=330 \mathrm{~km} \mathrm{~s}^{-1}\right)$ from Brott et al. (2011).

Figures 8 and 9 show the histograms of the relative frequency of our OB type sample located at low $\left(78.5^{\circ}-79.5^{\circ}\right)$, central $\left(79.5^{\circ}-80.5^{\circ}\right)$ and high $\left(80.5^{\circ}-81.5^{\circ}\right)$ Galactic longitudes in each age group and for each model considered (Geneva (Fig. 8) and Bonn (Fig. 9) rotating and non-rotating models). In all cases, most of the stars at high Galactic longitudes belong to the youngintermediate age group, while those stars located at low Galactic longitudes belong to the old age group. We only obtain significant age differences at using the rotating Geneva models since in this case rotation gives older ages for the most massive stars. Thus, we can see a change in the relative frequencies of old and intermediate stars. On the other hand, we do not see these age differences at using rotating Bonn models because of their lower sensitivity to rotation as noted earlier.

This analysis thus supports the correlation between ages and Galactic longitudes in Cygnus OB2 as previously suggested by Comerón \& Pasquali (2012). Massive star formation in Cygnus OB2 seems to have proceeded from lower to higher Galactic longitudes, regardless of the details of the models used.

\subsection{The whole Cygnus region}

In order to obtain a big picture of the age distribution across the Galactic longitude, we have performed the same analysis as in Cygnus OB2 but now in a wider area which includes Cygnus OB2, Cygnus OB9 and boundaries. We have placed the whole sample of OB stars, including late B-type stars, in an HRD (see Fig. 10). Due to the similar results obtained in Cygnus OB2 by using different models, we have now used isochrones and evolutionary stellar tracks for non-rotating models from Ekström et al. (2012). We derived ages by comparison with isochrones and divided again the sample in three age groups: the young group ( $0-5 \mathrm{Myr})$, the intermediate group (5-10 Myr), and the old group (>10 Myr). In Fig. 11 we show the spatial distribution of the different age groups, where most of the younger stars (left) are concentrated at higher Galactic longitudes, while the older ones (right) are located at central-lower Galactic longitudes. These results suggest that massive star formation has proceeded from lower to higher Galactic longitudes, 

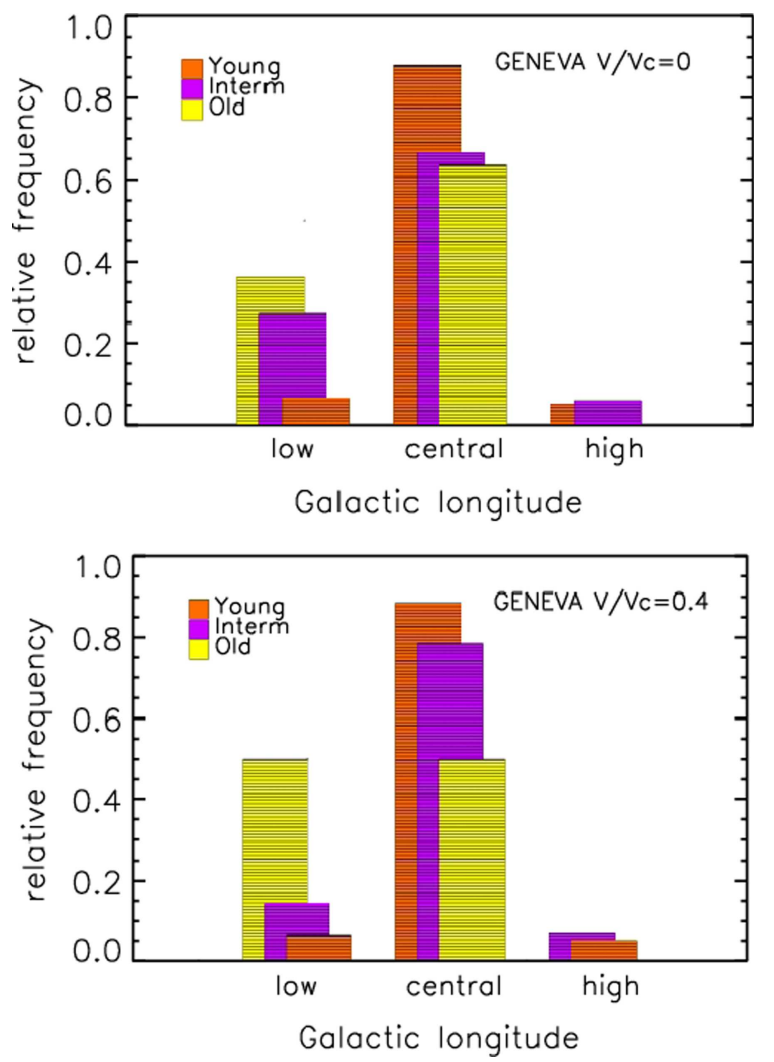

Fig. 8. Relative frequency histograms of the stars located at low $\left(78.5^{\circ}-\right.$ $\left.79.5^{\circ}\right)$, central $\left(79.5^{\circ}-80.5^{\circ}\right)$ and high $\left(80.5^{\circ}-81.5^{\circ}\right)$ Galactic longitudes in Cygnus OB2 using Geneva non-rotating (top) and rotating (bottom) models. Orange, purple and yellow colors indicate those stars located in the young ( $0-5 \mathrm{Myr})$, intermediate (5-10 Myr) and old (>10 Myr) age groups.

from Cygnus OB9 to Cygnus OB2, with a strong peak in the northern part of the association.

\section{Conclusions}

We have carried out several observing runs between 2013 and 2017 to obtain new blue intermediate-resolution spectra suitable for spectral classification for the magnitude-limited $(B \leq$ $16 \mathrm{mag}, K s<9 \mathrm{mag}$ ) candidate list compiled by Comerón \& Pasquali (2012). Out of 61 candidates, we confirm 42 new massive OB-type stars, earlier than B3, including 11 new O-type members. A $21 \%$ of this sample results on confirmed or possible SB2 binaries. Two other stars are late-B, seven are A-type, six are F-type, and the remaining four are G-type stars. Therefore, the spectral classification of the homogeneously selected sample is now completed. The selection criteria success rate obtained by Comerón \& Pasquali (2012) along with this work is $72 \%$, supporting the success at identifying reddened massive OB stars with the reddening-free parameters based on $B J H K$ photometry (see Sect. 2). However, the magnitude cutoff and dust extinction introduce an incompleteness. We loose the faintest and more obscured late O-type members in Cygnus OB2, and we are still far to obtain a complete census of the early-type population in the association.

We have also estimated individual visual extinctions $\left(A_{\mathrm{V}}\right)$ for the new confirmed OB-type stars using the extinction law derived by Wright et al. (2015). We found a median value of $\sim 5.5$ mag for the stars in the whole Cygnus region, and as we
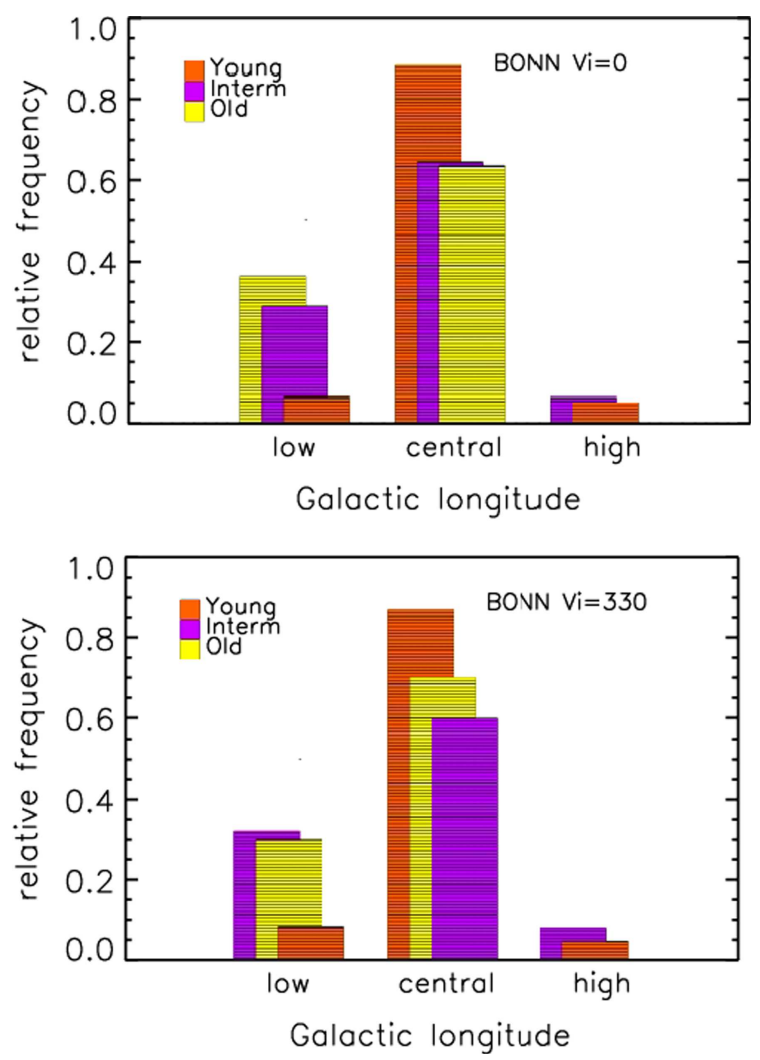

Fig. 9. Relative frequency histograms of the stars located at low $\left(78.5^{\circ}-\right.$ $\left.79.5^{\circ}\right)$, central $\left(79.5^{\circ}-80.5^{\circ}\right)$ and high $\left(80.5^{\circ}-81.5^{\circ}\right)$ Galactic longitudes in Cygnus OB2 using Bonn non-rotating (top) and rotating (bottom) models. Orange, purple and yellow colors indicate those stars located in the young (0-5 Myr), intermediate (5-10 Myr) and old (>10 Myr) age groups.

expect, a large median value of $\sim 6.5$ mag for those stars within the Cygnus OB2 association.

We have placed the new sample of OB stars in the HR diagram using both rotating and non-rotating models calculated by Ekström et al. (2012) and Brott et al. (2011) in order to assess uncertainties. We have also placed the already known OB type stars compiled by Comerón \& Pasquali (2012) for completeness. To this aim, we derived effective temperatures and luminosities using spectral type calibrations for the whole sample. Although coming from different sources, our adopted calibrations fit very well with each other. Uncertainties about rotation and adopted models affect only the most massive members, suggesting larger ages for them of $\sim 1-2$ Myr. Even so, all models support the previous age spread observed in Cygnus OB2 of 1-6 Myr.

In order to check the correlation between age and Galactic longitude found by Comerón \& Pasquali (2012) in Cygnus OB2, we have divided the sample of OB stars in different location and age groups by comparing with isochrones: the young group (0$5 \mathrm{Myr}$ ), the intermediate group (5-10 Myr), and the old group $(>10 \mathrm{Myr})$. Despite the differences in physical process treatment (rotation and physics of the stellar interior) done by the Geneva and Bonn groups, the spatial distribution of each age group is globally similar. Therefore, the result obtained by Comerón \& Pasquali (2012) can not be an effect of using the Geneva stellar models without rotation when age dating the Cygnus region stars. Most of the stars at high Galactic longitudes belong to the young-intermediate age group, while stars at low Galactic longitudes belong to the old group. Assuming a wider area which includes Cygnus OB2, part of Cygnus OB9 and boundaries, we 


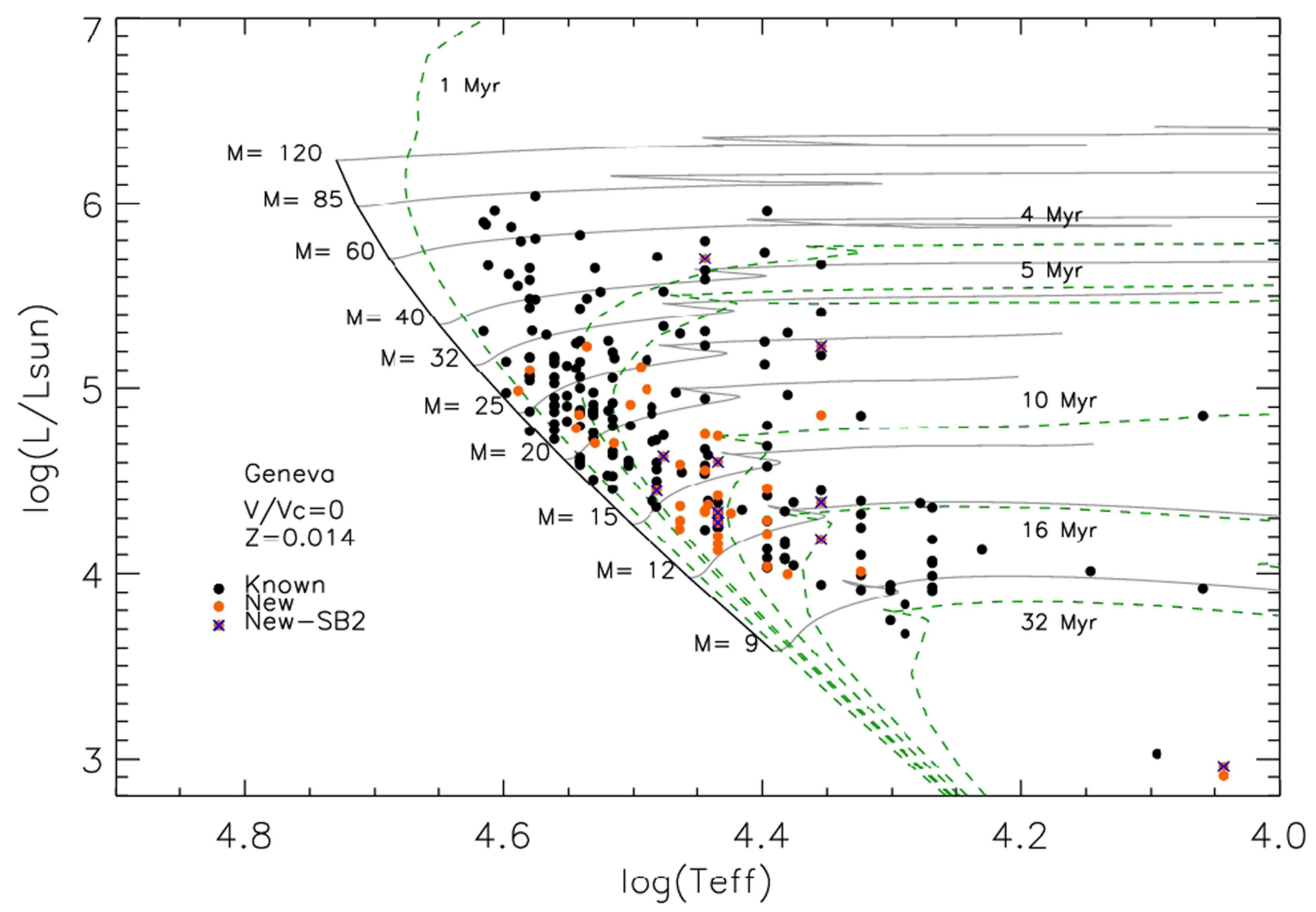

Fig. 10. HR diagram of OB stars in the Cygnus region (Cygnus OB2, Cygnus OB9 and field population) assuming a $D M=10.8$ mag. Known OB stars from Comerón \& Pasquali (2012) are also included to complete the sample. Black and orange dots indicate the already known and new OB-type stars respectively. Blue crosses indicate those new stars classified as possible or confirmed SB2 stars. Isochrones (dotted lines) and evolutionary stellar tracks (solid lines) for non-rotating models are from Ekström et al. (2012). Late-B stars are also included.
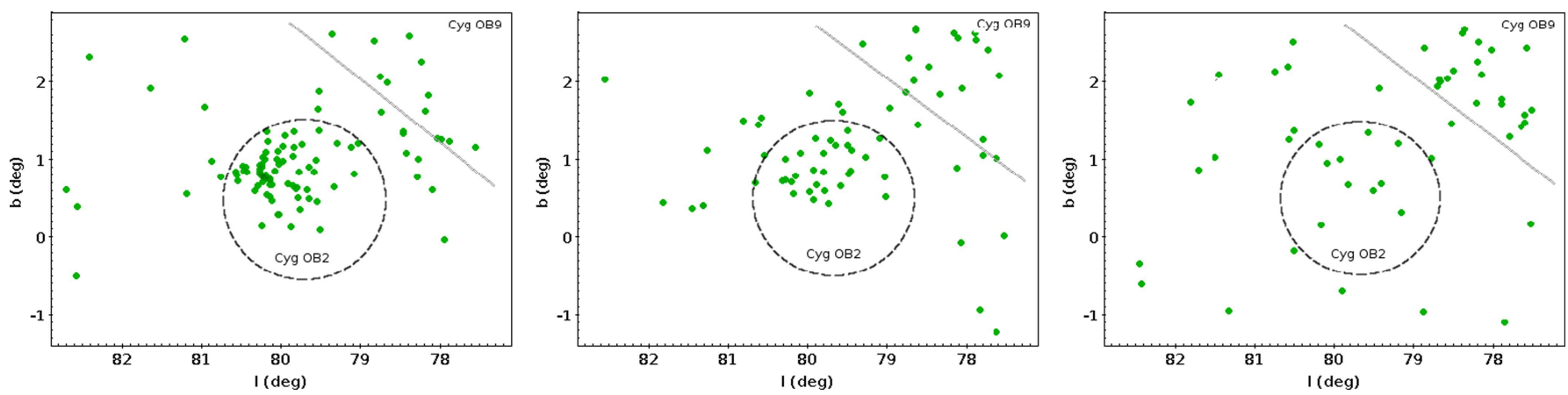

Fig. 11. Spatial distribution of stars of different age in the Cygnus area: the young population (0-5 Myr) on the left, the intermediate age one (5-10 Myr) in the middle, and the old population (>10 Myr) on the right.

obtain similar results suggesting that massive star formation in the region proceeds from Cygnus OB9 to Cygnus OB2, with a strong peak in the northern part of the association. Therefore the correlation between age and Galactic longitude is confirmed, regardless of the details of the models used.

Acknowledgements. We acknowledge financial support from the Spanish Ministry of Economy and Competitiveness (MINECO) under the grants AYA2012-39364-C02-01, AYA 2015-68012-C2-01 and Severo Ochoa SEV2015-0548. A.S. also acknowledges support from MINECO through grants AYA2013-40 611-P and AYA2016-75 931-C2-2-P. AP and CBM acknowledge support from the Sonderforschungsbereich SFB 881 "The Milky Way System" (subproject B5) of the German Research Foundation (DFG). We also thank the WHT and its service programme (SW2017a04), J. Maíz Apellániz for his useful MGB software, and N. Wright for helpful discussion and comments in this paper.

\section{References}

Brott, I., de Mink, S. E., Cantiello, M., et al. 2011, A\&A, 530, A115 Comerón, F., \& Pasquali, A. 2012, A\&A, 543, A101

Comerón, F., Pasquali, A., Rodighiero, G., et al. 2002, A\&A, 389, 874

Comerón, F., Pasquali, A., Figueras, F., \& Torra, J. 2008, A\&A, 486, 453

Comerón, F., Djupvik, A. A., Schneider, N., \& Pasquali, A. 2016, A\&A, 586, A46

Crowther, P. A., Lennon, D. J., \& Walborn, N. R. 2006, A\&A, 446, 279

Drew, J. E., Greimel, R., Irwin, M. J., \& Sale, S. E. 2008, MNRAS, 386, 1761

Ekström, S., Georgy, C., Eggenberger, P., et al. 2012, A\&A, 537, A146

Gray, R. O., \& Corbally, C. J. 2009, Stellar Spectral Classification (Princeton University Press)

Hanson, M. M. 2003, ApJ, 597, 957

Herrero, A., Corral, L. J., Villamariz, M. R., \& Martín, E. L. 1999, A\&A, 348, 542 
Herrero, A., Puls, J., Corral, L. J., Kudritzki, R. P., \& Villamariz, M. R. 2001, A\&A, 366, 623

Herrero, A., Puls, J., \& Najarro, F. 2002, A\&A, 396, 949

Humphreys, R. M., \& McElroy, D. B. 1984, ApJ, 284, 565

Kiminki, D. C., Kobulnicky, H. A., Kinemuchi, K., et al. 2007, ApJ, 664, 1102

Knödlseder, J. 2000, A\&A, 360, 539

Lanz, T., \& Hubeny, I. 2003, ApJS, 146, 417

Lanz, T., \& Hubeny, I. 2007, ApJS, 169, 83

Lejeune, T., \& Schaerer, D. 2001, A\&A, 366, 538

Maíz Apellániz, J., Pellerin, A., Barbá, R. H., et al. 2012, in Proc. Scientific

Meeting in Honor of Anthony, ed. F. J. Moffat, 465, 484

Maíz Apellániz, J., Sota, A., Arias, J. I., et al. 2016, ApJS, 224, 4

Markova, N., \& Puls, J. 2008, A\&A, 478, 823

Martins, F., \& Plez, B. 2006, A\&A, 457, 637

Martins, F., Schaerer, D., \& Hillier, D. J. 2005, A\&A, 436, 1049

Massey, P., \& Thompson, A. B. 1991, AJ, 101, 1408

Massey, P., Johnson, K. E., \& Degioia-Eastwood, K. 1995, ApJ, 454, 151

Massey, P., DeGioia-Eastwood, K., \& Waterhouse, E. 2001, AJ, 121, 1050

Morgan, W. W., Johnson, H. L., \& Roman, N. G. 1954, PASP, 66, 85

Münch, L., \& Morgan, W. W. 1953, ApJ, 118, 161

Negueruela, I., Marco, A., Herrero, A., \& Clark, J. S. 2008, A\&A, 487, 575

Nieva, M.-F. 2013, A\&A, 550, A26

Pecaut, M. J., \& Mamajek, E. E. 2013, ApJS, 208, 9

Portegies Zwart, S. F., McMillan, S. L. W., \& Gieles, M. 2010, ARA\&A, 48, 431
Reddish, V. C. 1968, The Observatory, 88, 139

Reipurth, B., \& Schneider, N. 2008, in Handbook of Star Forming Regions (ASP Monograph Publications), 4, 36

Rieke, G. H., \& Lebofsky, M. J. 1985, ApJ, 288, 618

Rygl, K. L. J., Brunthaler, A., Sanna, A., et al. 2012, A\&A, 539, A79

Sana, H., \& Evans, C. J. 2011, Active OB Stars: Structure, Evolution, Mass Loss, and Critical Limits, IAU Symp., 272, 474

Schneider, N., Bontemps, S., Simon, R., et al. 2006, A\&A, 458, 855

Schulte, D. H. 1956, ApJ, 124, 530

Schulte, D. H. 1958, ApJ, 128, 41

Simón-Díaz, S., \& Herrero, A. 2007, A\&A, 468, 1063

Simón-Díaz, S., Herrero, A., Sabín-Sanjulián, C., et al. 2014, A\&A, 570, L6

Simón-Díaz, S., Negueruela, I., Maíz Apellániz, J., et al. 2015, Highlights of Spanish Astrophysics VIII, 576

Skrutskie, M. F., Cutri, R. M., Stiening, R., et al. 2006, AJ, 131, 1163

Sota, A., Maíz Apellániz, J., Walborn, N. R., et al. 2011, ApJS, 193, 24

Tokunaga, A. T. 2000, Allen's Astrophysical Quantities, 143

Walborn, N. R. 1971, ApJS, 23, 257

Walborn, N. R. 1973, AJ, 78, 1067

Walborn, N. R., Howarth, I. D., Lennon, D. J., et al. 2002, AJ, 123, 2754

Wright, N. J., Drake, J. J., \& Drew, J. E. 2009, AAS Meet. Abstr., 213, 605.10

Wright, N. J., Drake, J. J., Drew, J. E., \& Vink, J. S. 2010, ApJ, 713, 871

Wright, N. J., Drew, J. E., \& Mohr-Smith, M. 2015, MNRAS, 449, 741

Zacharias, N., Urban, S. E., Zacharias, M. I., et al. 2004, AJ, 127, 3043

Zacharias, N., Finch, C., Girard, T., et al. 2010, AJ, 139, 2184 


\section{Appendix A: Table of stellar parameters}

Table A.1 shows the derived stellar parameters for the new classified OB stars in a large area which includes Cygnus OB2 and its surroundings. We have also included the derived individual visual extinctions. Table A.2 shows the updated temperatures and luminosities for the already known OB stars from Comerón \& Pasquali (2012) assuming the same spectral calibrations used in this work (see Sect. 4.2).

Table A.1. Temperatures, luminosities, and individual visual extinctions derived for the new classified massive OB stars.

\begin{tabular}{|c|c|c|c|c|}
\hline Object & $\mathrm{SpT}$ & $T_{\text {eff }}(\mathrm{K})$ & $\log \left(L / L_{\odot}\right)$ & $A_{\mathrm{V}}(\mathrm{mag})$ \\
\hline \multicolumn{5}{|l|}{ Cygnus $O B 2$} \\
\hline $\mathrm{J} 20345785+4143543$ & O7:Ib & 34990 & 5.25 & 8.2 \\
\hline J20293563+4024315 & O8IIIz & 33961 & 4.75 & 5.1 \\
\hline J20275292+4144067 & O9.5II & 30626 & 5.09 & 7.1 \\
\hline J20291617+4057372 & O9.7III & 28518 & 4.78 & 7.7 \\
\hline $\mathrm{J} 20273787+4115468$ & B0II & 25932 & 4.62 & 6.8 \\
\hline $\mathrm{J} 20301097+4120088$ & B0:II: & 25932 & 4.39 & 7.4 \\
\hline $\mathrm{J} 20323968+4050418$ & B0II & 25932 & 4.32 & 5.7 \\
\hline $\mathrm{J} 20323882+4058469$ & B0Ib & 27800 & 4.33 & 6.7 \\
\hline $\mathrm{J} 20272099+4121262$ & B0.5V & 26308 & 4.33 & 5.8 \\
\hline $\mathrm{J} 20330526+4143367$ & B0.5III & 24164 & 4.27 & 5.2 \\
\hline BD+404208 & B1V & 23590 & 4.14 & 2.2 \\
\hline $\mathrm{J} 20314341+4100021$ & $\mathrm{~B} 1 \mathrm{~V}$ & 23590 & 4.21 & 7.0 \\
\hline $\mathrm{J} 20315898+4107314$ & B1V & 23590 & 4.26 & 7.1 \\
\hline $\mathrm{BD}+404193$ & B1V & 23590 & 4.06 & 1.7 \\
\hline $\mathrm{J} 20274925+4017004$ & B1III & 21557 & 4.27 & 4.9 \\
\hline $\mathrm{J} 20315433+4010067$ & B1III & 21557 & 4.02 & 6.5 \\
\hline CCDMJ20323+4152AB & B9V & 10700 & 2.96 & -0.2 \\
\hline \multicolumn{5}{|l|}{ Surroundings } \\
\hline $\mathrm{J} 20423509+4256364$ & O6IIIz & 38192 & 4.97 & 7.4 \\
\hline J20371773+4156316 & $\mathrm{O} 7 \mathrm{~V}$ & 36872 & 5.06 & 8.2 \\
\hline $\mathrm{J} 20222481+4013426$ & O8II & 33570 & 4.69 & 5.5 \\
\hline $\mathrm{J} 20261976+3951425$ & O8.5IV & 33391 & 4.81 & 8.0 \\
\hline $\mathrm{J} 20262484+4001413$ & O9.2III & 31317 & 4.65 & 5.9 \\
\hline J20382173+4157069 & O9.7II & 30859 & 4.99 & 8.5 \\
\hline $\mathrm{J} 20181090+4029063$ & 09.7Ib & 28644 & 4.58 & 7.6 \\
\hline $\mathrm{J} 20395358+4222506$ & B0I & 25094 & 5.57 & 11.0 \\
\hline J20281176+3840227 & B0Ib & 25094 & 4.43 & 3.6 \\
\hline $\mathrm{J} 20290247+4231159$ & $\mathrm{~B} 1 \mathrm{Ib}$ & 19979 & 4.03 & 5.0 \\
\hline $\mathrm{J} 20225451+4023314$ & B0Iab & 25094 & 4.21 & 4.7 \\
\hline $\mathrm{J} 20253320+4048444$ & B0Iab & 25094 & 4.62 & 5.5 \\
\hline HDE229258 & $\mathrm{B} 0.7 \mathrm{~V}$ & 24949 & 4.13 & 1.8 \\
\hline $\mathrm{J} 20361806+4228483$ & B0.7III & 22860 & 4.14 & 7.2 \\
\hline J20233816+3938118 & $\mathrm{B} 0.7 \mathrm{Ib}$ & 21514 & 3.87 & 2.3 \\
\hline HD 228973 & $\mathrm{~B} 1 \mathrm{~V}$ & 23590 & 4.45 & 2.4 \\
\hline $\mathrm{J} 20201435+4107155$ & $\mathrm{~B} 1 \mathrm{~V}$ & 23590 & 4.27 & 3.4 \\
\hline $\mathrm{J} 20230290+4133466$ & $\mathrm{~B} 1 \mathrm{~V}$ & 23590 & 4.68 & 7.2 \\
\hline $\mathrm{J} 20330453+3822269$ & B1V & 23590 & 4.09 & 2.4 \\
\hline $\mathrm{J} 20230183+4014029$ & B1III & 21557 & 4.26 & 4.3 \\
\hline $\mathrm{J} 20382889+4009566$ & B1III & 21557 & 3.84 & 1.4 \\
\hline $\mathrm{J} 20440752+4107342$ & B1III & 21557 & 4.09 & 6.5 \\
\hline LSII+3797 & B1Ia & 19979 & 5.07 & 5.8 \\
\hline $\mathrm{J} 20211924+3936230$ & $\mathrm{~B} 1 \mathrm{Ib}$ & 19979 & 4.23 & 4.6 \\
\hline $\mathrm{BD}+394179$ & $\mathrm{~B} 1 \mathrm{Ib}$ & 19979 & 4.70 & 4.2 \\
\hline $\mathrm{J} 20381289+4057169$ & $\mathrm{~B} 2 \mathrm{~V}$ & 20549 & 3.98 & 4.2 \\
\hline $\mathrm{BD}+423785 \mathrm{a}$ & B9V & 10700 & 2.90 & 0.6 \\
\hline
\end{tabular}


A\&A 612, A50 (2018)

Table A.2. Temperatures and luminosities derived for the known massive OB stars from Comerón \& Pasquali (2012).

\begin{tabular}{|c|c|c|c|c|c|}
\hline Object & RA (hhmmss) & $\operatorname{Dec}\left({ }^{\circ} \prime^{\prime \prime}\right)$ & $\mathrm{SpT}$ & $T_{\text {eff }}(\mathrm{K})$ & $\log \left(L / L_{\odot}\right)$ \\
\hline \multicolumn{6}{|l|}{ Cygnus OB2 } \\
\hline $\mathrm{J} 20331411+4120218$ & 20:33:14.110 & $+41: 20: 21.91$ & O3If & 40910 & 5.66 \\
\hline J20330879+4113179 & 20:33:08.818 & $+41: 13: 17.93$ & O4III & 41070 & 5.88 \\
\hline $\mathrm{J} 20360451+4056129$ & $20: 36: 04.500$ & $+40: 56: 13.01$ & $\mathrm{O} 5 \mathrm{~V}((\mathrm{f}))$ & 41250 & 5.31 \\
\hline J20331798+4118311 & 20:33:17.982 & $+41: 18: 31.19$ & O5III & 39440 & 5.62 \\
\hline $\mathrm{J} 20340850+4136592$ & 20:34:08.514 & $+41: 36: 59.39$ & O5I & 37630 & 5.80 \\
\hline J20331074+4115081 & $20: 33: 10.735$ & $+41: 15: 08.22$ & O5If & 37630 & 6.04 \\
\hline $\mathrm{J} 20332346+4109130$ & $20: 33: 23.471$ & $+41: 09: 12.90$ & $\mathrm{O} 5.5 \mathrm{~V}$ & 40440 & 5.96 \\
\hline J20331326+4113287 & $20: 33: 13.264$ & $+41: 13: 28.67$ & O6V & 39630 & 5.14 \\
\hline J20303980+4136506 & $20: 30: 39.805$ & $+41: 36: 50.63$ & O6V & 39630 & 4.97 \\
\hline $\mathrm{J} 20344410+4051584$ & $20: 34: 44.146$ & $+40: 51: 58.67$ & O6.5III(f) & 37845 & 5.31 \\
\hline J20283203+4049027 & $20: 28: 32.027$ & $+40: 49: 02.88$ & $\mathrm{O} 7$ & 38010 & 5.65 \\
\hline J20310019+4049497 & 20:31:00.204 & $+40: 49: 49.70$ & $\mathrm{O} 7 \mathrm{~V}((\mathrm{f}))$ & 38010 & 5.06 \\
\hline $\mathrm{J} 20341350+4135027$ & 20:34:13.511 & $+41: 35: 02.86$ & $\mathrm{O} 7 \mathrm{~V}$ & 38010 & 5.04 \\
\hline $\mathrm{J} 20331748+4117093$ & $20: 33: 17.483$ & $+41: 17: 09.35$ & $\mathrm{O} 7 \mathrm{~V}$ & 38010 & 5.17 \\
\hline J20334086+4130189 & $20: 33: 40.863$ & $+41: 30: 18.95$ & O7V & 38010 & 4.77 \\
\hline $\mathrm{J} 20342959+4131455$ & 20:34:29.599 & $+41: 31: 45.49$ & $\mathrm{O} 7 \mathrm{~V}$ & 38010 & 5.58 \\
\hline J20315961+4114505 & 20:31:59.609 & $+41: 14: 50.45$ & $\mathrm{O} 7 \mathrm{~V}$ & 38010 & 4.87 \\
\hline $\mathrm{J} 20321383+4127120$ & $20: 32: 13.822$ & $+41: 27: 12.01$ & O7IIIf & 36900 & 5.29 \\
\hline $\mathrm{J} 20313690+4059092$ & $20: 31: 36.911$ & $+40: 59: 09.06$ & $\mathrm{O} 7 \mathrm{Ib}(\mathrm{f})$ & 34350 & 5.48 \\
\hline J20323154+4114082 & 20:32:31.531 & $+41: 14: 08.18$ & O7.5Ib-II(f) & 33530 & 5.52 \\
\hline $\mathrm{J} 20323857+4125137$ & $20: 32: 38.571$ & $+41: 25: 13.79$ & $\mathrm{O} 8 \mathrm{~V}(\mathrm{n})$ & 36390 & 5.02 \\
\hline $\mathrm{J} 20274361+4035435$ & $20: 27: 43.616$ & $+40: 35: 43.51$ & O8V: & 36390 & 4.72 \\
\hline $\mathrm{J} 20331369+4113057$ & $20: 33: 13.688$ & $+41: 13: 05.77$ & $\mathrm{O} 8 \mathrm{~V}$ & 36390 & 4.87 \\
\hline J20324545+4125374 & $20: 32: 45.450$ & $+41: 25: 37.57$ & $\mathrm{O} 8 \mathrm{~V}$ & 36390 & 5.16 \\
\hline J20331803+4121366 & $20: 33: 18.035$ & $+41: 21: 36.67$ & $\mathrm{O} 8 \mathrm{~V}$ & 36390 & 4.90 \\
\hline $\mathrm{J} 20323486+4056174$ & $20: 32: 34.865$ & $+40: 56: 17.35$ & $\mathrm{O} 8 \mathrm{~V}$ & 36390 & 4.77 \\
\hline $\mathrm{J} 20300788+4123504$ & 20:30:07.877 & $+41: 23: 50.44$ & $\mathrm{O} 8 \mathrm{~V}$ & 36390 & 4.91 \\
\hline $\mathrm{J} 20330292+4117431$ & 20:33:02.913 & $+41: 17: 43.16$ & $\mathrm{O} 8 \mathrm{~V}$ & 36390 & 5.06 \\
\hline $\mathrm{J} 20325002+4123446$ & $20: 32: 50.016$ & $+41: 23: 44.70$ & $\mathrm{O} 8 \mathrm{~V}$ & 36390 & 4.95 \\
\hline $\mathrm{J} 20325919+4124254$ & $20: 32: 59.057$ & $+41: 24: 24.79$ & $\mathrm{O} 8 \mathrm{~V}$ & 36390 & 4.81 \\
\hline $\mathrm{J} 20333030+4135578$ & $20: 33: 30.316$ & $+41: 35: 57.88$ & O8V & 36390 & 5.17 \\
\hline J20342193+4117016 & $20: 34: 21.934$ & $+41: 17: 01.66$ & $\mathrm{O} 8 \mathrm{III}+\mathrm{O} 8 \mathrm{III}$ & 35010 & 5.11 \\
\hline $\mathrm{J} 20323843+4040445$ & $20: 32: 38.441$ & $+40: 40: 44.48$ & O8III & 35010 & 5.24 \\
\hline $\mathrm{J} 20330292+4047254$ & 20:33:02.928 & $+40: 47: 25.29$ & O8II((f)) & 33860 & 5.65 \\
\hline $\mathrm{J} 20314540+4118267$ & $20: 31: 45.403$ & $+41: 18: 26.73$ & O8I & 32710 & 5.16 \\
\hline J30332557+4133269 & $20: 33: 25.569$ & $+41: 33: 26.88$ & $08.5 \mathrm{~V}$ & 35580 & 5.12 \\
\hline J20331634+4119017 & $20: 33: 16.256$ & $+41: 19: 00.16$ & $08.5 \mathrm{~V}$ & 35580 & 4.82 \\
\hline J20332674+4110595 & $20: 33: 26.756$ & $+41: 10: 59.42$ & $08.5 \mathrm{~V}$ & 35580 & 4.90 \\
\hline $\mathrm{J} 20313749+4113210$ & $20: 31: 37.506$ & $+41: 13: 20.99$ & O9: & 34770 & 5.83 \\
\hline J20335842+4019411 & $20: 33: 58.417$ & $+40: 19: 41.13$ & O9: & 34770 & 5.43 \\
\hline J20321656+4125357 & $20: 32: 16.563$ & $+41: 25: 35.67$ & $\mathrm{O} 9 \mathrm{~V}$ & 34770 & 4.79 \\
\hline $\mathrm{J} 20311833+4121216$ & $20: 31: 18.329$ & $+41: 21: 21.65$ & $\mathrm{O} 9 \mathrm{~V}$ & 34770 & 5.06 \\
\hline $\mathrm{J} 20340486+4105129$ & 20:34:04.851 & $+41: 05: 11.76$ & O9V & 34770 & 4.59 \\
\hline $\mathrm{J} 20311055+4131535$ & $20: 31: 10.543$ & $+41: 31: 53.53$ & $\mathrm{O} 9 \mathrm{~V}$ & 34770 & 5.14 \\
\hline J20332101+4117401 & $20: 33: 21.016$ & $+41: 17: 40.11$ & O9V & 34770 & 4.63 \\
\hline $\mathrm{J} 20331571+4120172$ & $20: 33: 15.685$ & $+41: 20: 18.75$ & O9V & 34770 & 4.60 \\
\hline $\mathrm{J} 20301839+4053466$ & $20: 30: 18.391$ & $+40: 53: 46.56$ & $\mathrm{O} 9 \mathrm{~V}$ & 34770 & 4.99 \\
\hline J20314965+4128265 & $20: 31: 49.658$ & $+41: 28: 26.50$ & O9III & 33120 & 4.53 \\
\hline $\mathrm{J} 20345606+4038179$ & $20: 34: 56.057$ & $+40: 38: 17.92$ & O9.7Iab & 29922 & 5.33 \\
\hline J20305772+4109575 & $20: 30: 57.727$ & $+41: 09: 57.51$ & $09.5 \mathrm{~V}$ & 33960 & 4.85 \\
\hline $\mathrm{J} 20340601+4108090$ & 20:34:06.017 & $+41: 08: 09.13$ & $09.5 \mathrm{~V}$ & 33960 & 4.87 \\
\hline $\mathrm{J} 20335952+4117354$ & $20: 33: 59.527$ & $+41: 17: 35.46$ & $09.5 \mathrm{~V}$ & 33960 & 4.91 \\
\hline $\mathrm{J} 20341605+4102196$ & $20: 34: 16.046$ & $+41: 02: 19.59$ & $09.5 \mathrm{~V}$ & 33960 & 4.73 \\
\hline $\mathrm{J} 20272428+4115458$ & $20: 27: 24.282$ & $+41: 15: 45.82$ & $09.5 \mathrm{~V}$ & 33960 & 4.51 \\
\hline $\mathrm{J} 20293480+4120089$ & 20:29:34.798 & $+41: 20: 08.93$ & $09.5 \mathrm{~V}$ & 33960 & 4.76 \\
\hline $\mathrm{J} 20323033+4034332$ & $20: 32: 30.310$ & $+40: 34: 33.22$ & O9.5IV & 33067 & 5.25 \\
\hline $\mathrm{J} 20333700+4116113$ & $20: 33: 36.994$ & $+41: 16: 11.31$ & O9.5IV & 33067 & 4.88 \\
\hline $\mathrm{J} 20334610+4133010$ & 20:33:46.112 & $+41: 33: 01.00$ & O9.5Ia & 30250 & 5.71 \\
\hline J20325964+4115146 & $20: 32: 59.633$ & $+41: 15: 14.66$ & O9.7III & 31797 & 4.80 \\
\hline $\mathrm{J} 20283039+4105290$ & $20: 28: 30.385$ & $+41: 05: 29.04$ & OC9.7Ia & 29922 & 5.52 \\
\hline
\end{tabular}


S. R. Berlanas et al.: New massive members of Cygnus OB2

Table A.2. continued.

\begin{tabular}{|c|c|c|c|c|c|}
\hline Object & RA (hhmmss) & $\operatorname{Dec}\left({ }^{\circ} \prime \prime \prime\right)$ & $\mathrm{SpT}$ & $T_{\text {eff }}(\mathrm{K})$ & $\log \left(L / L_{\odot}\right)$ \\
\hline $\mathrm{J} 20302730+4113253$ & 20:30:27.300 & $+41: 13: 25.13$ & Ofpe & 38612 & 5.79 \\
\hline $\mathrm{J} 20281547+4038196$ & $20: 28: 15.471$ & $+40: 38: 19.81$ & B0V: & 32816 & 4.79 \\
\hline $\mathrm{J} 20323951+4052475$ & $20: 32: 39.507$ & $+40: 52: 47.46$ & B0:V: & 32816 & 5.19 \\
\hline $\mathrm{J} 20331050+4122224$ & $20: 33: 10.502$ & $+41: 22: 22.44$ & B0V & 32816 & 4.45 \\
\hline J20305552+4109575 & $20: 30: 55.516$ & $+40: 54: 54.03$ & B0V & 32816 & 4.66 \\
\hline $\mathrm{J} 20295701+4109538$ & $20: 29: 57.010$ & $+41: 09: 53.84$ & BOV & 32816 & 4.64 \\
\hline $\mathrm{J} 20305111+4120218$ & 20:30:51.115 & $+41: 20: 21.78$ & B0V & 32816 & 4.53 \\
\hline $\mathrm{J} 20331130+4042337$ & 20:33:11.300 & $+40: 42: 33.73$ & B0:III: & 30308 & 4.72 \\
\hline $\mathrm{J} 20333821+4041064$ & $20: 33: 38.213$ & $+40: 41: 06.35$ & B0Ia & 27800 & 5.64 \\
\hline $\mathrm{J} 20344471+4051465$ & $20: 34: 44.716$ & $+40: 51: 46.73$ & B0Ia & 27800 & 5.59 \\
\hline $\mathrm{J} 20323904+4100078$ & $20: 32: 39.057$ & $+41: 00: 07.78$ & B0Ia & 27800 & 5.79 \\
\hline J20322774+4128522 & $20: 32: 27.738$ & $+41: 28: 52.26$ & B0Ib & 27800 & 4.24 \\
\hline J20345878+4136174 & 20:34:58.781 & $+41: 36: 17.35$ & $\mathrm{~B} 0 \mathrm{Ib}(\mathrm{n}) \mathrm{sb}$ & 27800 & 5.31 \\
\hline $\mathrm{J} 20333822+4053412$ & $20: 33: 38.218$ & $+40: 53: 41.19$ & B0Ib & 27800 & 4.94 \\
\hline $\mathrm{J} 20333910+4119258$ & $20: 33: 39.102$ & $+41: 19: 25.98$ & B0Iab & 27800 & 5.23 \\
\hline $\mathrm{J} 20323498+4052390$ & $20: 32: 34.848$ & $+40: 52: 39.46$ & $\mathrm{~B} 0.2 \mathrm{~V}$ & 31906 & 4.58 \\
\hline $\mathrm{J} 20292449+4052599$ & $20: 29: 24.485$ & $+40: 52: 59.85$ & B0.2IV & 30588 & 4.71 \\
\hline $\mathrm{J} 20321568+4046170$ & $20: 32: 15.679$ & $+40: 46: 17.00$ & B0.2IV & 30588 & 4.39 \\
\hline J20294666+4105083 & $20: 29: 46.672$ & $+41: 05: 08.32$ & $\mathrm{~B} 0.5 \mathrm{~V}(\mathrm{n}) \mathrm{sb}$ & 30288 & 4.50 \\
\hline $\mathrm{J} 20282772+4104018$ & $20: 28: 27.723$ & $+41: 04: 01.80$ & $\mathrm{~B} 0.5 \mathrm{~V}$ & 30288 & 4.47 \\
\hline J20314605+4043246 & $20: 31: 46.053$ & $+40: 43: 24.61$ & B0.5IV & 28969 & 4.55 \\
\hline $\mathrm{J} 20331870+4059379$ & 20:33:18.696 & $+40: 59: 37.92$ & B0.5IIIe & 27651 & 4.64 \\
\hline J20303970+4108489 & $20: 30: 39.701$ & $+41: 08: 48.80$ & $\mathrm{~B} 0.7 \mathrm{Ib}$ & 24014 & 5.30 \\
\hline $\mathrm{J} 20294060+4109585$ & $20: 29: 40.601$ & $+41: 09: 58.54$ & $\mathrm{~B} 1[\mathrm{e}]$ & 27173 & 4.27 \\
\hline J20313338+4122490 & $20: 31: 33.378$ & $+41: 22: 49.02$ & B1V & 27173 & 4.25 \\
\hline $\mathrm{J} 20340435+4108078$ & 20:34:04.349 & $+41: 08: 07.91$ & $\mathrm{~B} 1 \mathrm{~V}$ & 27173 & 4.27 \\
\hline $\mathrm{J} 20303833+4010538$ & $20: 30: 38.329$ & $+40: 10: 53.84$ & B1V & 27173 & 4.34 \\
\hline J20293473+4020381 & $20: 29: 34.728$ & $+40: 20: 38.09$ & $\mathrm{~B} 1 \mathrm{~V}$ & 27173 & 4.38 \\
\hline $\mathrm{J} 20273982+4040384$ & $20: 27: 39.821$ & $+40: 40: 38.35$ & $\mathrm{~B} 1 \mathrm{~V}$ & 27173 & 4.28 \\
\hline J20303297+4044024 & $20: 30: 32.965$ & $+40: 44: 02.41$ & B1V & 27173 & 4.31 \\
\hline $\mathrm{J} 20310464+4030568$ & 20:31:04.659 & $+40: 30: 56.93$ & B1III:e & 24903 & 5.95 \\
\hline J20334783+4120415 & $20: 33: 47.831$ & $+41: 20: 41.37$ & B1III & 24903 & 4.80 \\
\hline $\mathrm{J} 20281539+4044046$ & $20: 28: 15.392$ & $+40: 44: 04.57$ & B1III & 24903 & 4.58 \\
\hline $\mathrm{J} 20310700+4035537$ & 20:31:07.003 & $+40: 35: 53.73$ & B1III & 24903 & 4.08 \\
\hline J20314885+4038001 & $20: 31: 48.848$ & $+40: 38: 00.05$ & B1II & 23767 & 4.04 \\
\hline $\mathrm{J} 20333078+4115226$ & $20: 33: 30.791$ & $+41: 15: 22.70$ & B1I & 22632 & 5.41 \\
\hline J20312203+4131284 & $20: 31: 22.026$ & $+41: 31: 28.40$ & B1Ib: & 22632 & 4.45 \\
\hline J20322734+4055184 & $20: 32: 27.339$ & $+40: 55: 18.25$ & $\mathrm{~B} 2 \mathrm{~V}$ & 21092 & 4.39 \\
\hline J20312210+4112029 & $20: 31: 22.101$ & $+41: 12: 02.87$ & $\mathrm{~B} 2 \mathrm{~V}$ & 21092 & 4.10 \\
\hline $\mathrm{J} 20354703+4053012$ & $20: 35: 47.026$ & $+40: 53: 01.17$ & $\mathrm{~B} 2 \mathrm{~V}$ & 21092 & 3.91 \\
\hline $\mathrm{J} 20284657+4107069$ & $20: 28: 46.566$ & $+41: 07: 06.86$ & B2II & 19482 & 3.83 \\
\hline $\mathrm{J} 20320689+4117570$ & 20:32:06.877 & $+41: 17: 56.97$ & B3 V & 18546 & 3.91 \\
\hline \multicolumn{6}{|l|}{ Cygnus OB9 } \\
\hline HD 229196 & $20: 23: 10.784$ & $+40: 52: 29.85$ & O5 & 41250 & 5.89 \\
\hline $\mathrm{J} 20223777+4140292$ & $20: 22: 37.766$ & $+41: 40: 29.23$ & O5If & 37630 & 5.48 \\
\hline $\mathrm{BD}+394177$ & $20: 25: 22.122$ & $+40: 13: 01.09$ & O6.5 & 38820 & 5.55 \\
\hline HD 229250 & $20: 24: 11.733$ & $+39: 40: 41.54$ & $\mathrm{O} 7$ & 38010 & 5.44 \\
\hline $\mathrm{BD}+394168$ & $20: 24: 21.475$ & $+39: 46: 03.90$ & $\mathrm{O} 7$ & 38010 & 5.48 \\
\hline HD 229202 & $20: 23: 22.840$ & $+40: 09: 22.53$ & O8V: & 36390 & 5.14 \\
\hline $\mathrm{BD}+404159$ & $20: 25: 06.521$ & $+40: 35: 49.78$ & O9V & 34770 & 4.61 \\
\hline $\mathrm{BD}+404148$ & $20: 23: 14.549$ & $+40: 45: 19.07$ & $09.5: \mathrm{V}$ & 33960 & 4.88 \\
\hline J20194916+4052090 & 20:19:49.156 & $+40: 52: 08.99$ & $09.5 \mathrm{~V}$ & 33960 & 4.86 \\
\hline J20190610+4037004 & 20:19:06.102 & $+40: 37: 00.39$ & O9.7Iab & 29922 & 4.74 \\
\hline HD 193945 & $20: 21: 25.823$ & $+41: 11: 39.56$ & BoVnn & 32816 & 4.92 \\
\hline $\mathrm{BD}+384058$ & $20: 23: 28.531$ & $+39: 20: 59.05$ & BoV & 32816 & 4.83 \\
\hline LSII+4032 & $20: 25: 28.893$ & $+40: 12: 54.13$ & BOIII & 30308 & 4.36 \\
\hline J20243872+3930301 & $20: 24: 38.720$ & $+39: 30: 30.10$ & B0I: & 27800 & 4.58 \\
\hline $\mathrm{J} 20183413+4025045$ & $20: 18: 34.130$ & $+40: 25: 04.47$ & B0.2IV & 30588 & 4.86 \\
\hline NGC6910-14 & 20:23:07.575 & $+40: 46: 08.87$ & $\mathrm{~B} 0.5 \mathrm{~V}$ & 30288 & 4.56 \\
\hline $\mathrm{J} 20240515+4046035$ & $20: 24: 05.154$ & $+40: 46: 03.51$ & $\mathrm{~B} 0.5 \mathrm{~V}$ & 30288 & 4.60 \\
\hline
\end{tabular}


A\&A 612, A50 (2018)

Table A.2. continued.

\begin{tabular}{|c|c|c|c|c|c|}
\hline Object & RA (hhmmss) & $\operatorname{Dec}\left({ }^{\circ} \prime \prime \prime\right)$ & SpT & $T_{\text {eff }}(\mathrm{K})$ & $\log \left(L / L_{\odot}\right)$ \\
\hline HD 194092 & $20: 22: 05.443$ & $+40: 59: 08.17$ & B0.5III & 27651 & 4.39 \\
\hline HD 228882 & 20:18:57.784 & $+40: 42: 18.52$ & B0.5Ia & 25014 & 5.25 \\
\hline HD 228929 & $20: 19: 36.542$ & $+39: 54: 41.80$ & $\mathrm{~B} 0.5 \mathrm{Ib}$ & 25014 & 5.13 \\
\hline J20234624+3937078 & $20: 23: 46.238$ & $+39: 37: 07.83$ & B0.7IV & 27818 & 4.54 \\
\hline J20241767+3920326 & 20:24:17.666 & $+39: 20: 32.56$ & B1V & 27173 & 4.25 \\
\hline J20214868+4043005 & $20: 21: 48.682$ & $+40: 43: 00.45$ & $\mathrm{~B} 1 \mathrm{~V}$ & 27173 & 4.32 \\
\hline HD 228919 & 20:19:27.908 & $+40: 27: 42.09$ & B1IV & 26038 & 4.34 \\
\hline J20233375+4045199 & $20: 23: 33.752$ & $+40: 45: 19.93$ & B1III & 24903 & 4.03 \\
\hline J20223944+3935420 & $20: 22: 39.442$ & $+39: 35: 42.02$ & B1III & 24903 & 4.42 \\
\hline J20220454+4042487 & 20:22:04.541 & $+40: 42: 48.73$ & B1III & 24903 & 4.28 \\
\hline J20215593+4110129 & 20:21:55.930 & $+41: 10: 12.92$ & B1III & 24903 & 4.03 \\
\hline $\mathrm{J} 20220879+3958161$ & $20: 22: 08.793$ & $+39: 58: 16.07$ & B1II & 23767 & 4.38 \\
\hline $\mathrm{J} 20214410+4012529$ & 20:21:44.103 & $+40: 12: 52.91$ & B1Ia & 22632 & 5.18 \\
\hline J20215160+3959496 & 20:21:51.600 & $+39: 59: 49.61$ & $\mathrm{~B} 1 \mathrm{Ib}$ & 22632 & 3.94 \\
\hline J20203933+4031176 & $20: 20: 39.334$ & $+40: 31: 17.64$ & $\mathrm{~B} 1.5 \mathrm{~V}$ & 24132 & 4.08 \\
\hline J20204933+4033027 & $20: 20: 49.333$ & $+40: 33: 02.73$ & $\mathrm{~B} 1.5 \mathrm{~V}$ & 24132 & 4.15 \\
\hline HD 228911 & $20: 19: 21.712$ & $+40: 53: 16.46$ & B2 & 21092 & 4.25 \\
\hline HD 194194 & $20: 22: 44.760$ & $+40: 42: 52.63$ & B2III & 20019 & 3.91 \\
\hline J20211677+4023162 & $20: 21: 16.773$ & $+40: 23: 16.19$ & B2III & 20019 & 3.94 \\
\hline $\mathrm{J} 20250591+4020124$ & 20:25:05.912 & $+40: 20: 12.44$ & B2III & 20019 & 3.75 \\
\hline HD 228928 & 20:19:32.709 & $+40: 39: 13.75$ & B2Ib:nn & 18945 & 4.38 \\
\hline HD 228941 & 20:19:40.169 & $+40: 53: 19.19$ & B3 & 18546 & 3.98 \\
\hline $\mathrm{BD}+404146$ & $20: 23: 10.464$ & $+40: 45: 52.34$ & B3 & 18546 & 4.35 \\
\hline NGC6910-16 & 20:23:07.301 & $+40: 46: 55.25$ & B3 & 18546 & 4.05 \\
\hline $\mathrm{J} 20215115+3934215$ & $20: 21: 51.149$ & $+39: 37: 51.47$ & $\mathrm{~B} 3 \mathrm{~V}$ & 18546 & 4.18 \\
\hline J20221729+3946035 & $20: 22: 17.286$ & $+39: 34: 21.50$ & B5Ia & 14012 & 4.01 \\
\hline HD 228821 & 20:18:04.930 & $+40: 06: 06.80$ & B8 & 12449 & 3.03 \\
\hline HD 193426 & 20:18:39.749 & $+40: 13: 36.89$ & B9Ia & 11457 & 4.85 \\
\hline \multicolumn{6}{|l|}{ Boundaries } \\
\hline $\mathrm{BD}+433654$ & $20: 33: 36.079$ & $+43: 59: 07.38$ & O4If & 39270 & 5.87 \\
\hline LSII+3953 & 20:27:17.572 & $+39: 44: 32.60$ & O7V: & 38010 & 5.07 \\
\hline $\mathrm{BD}+423760$ & $20: 28: 40.812$ & $+43: 08: 58.46$ & $08.5 \mathrm{~V}$ & 35580 & 4.96 \\
\hline $\mathrm{BD}+423835$ & $20: 42: 06.863$ & $+43: 11: 03.72$ & O9p... & 34770 & 5.25 \\
\hline J20342894+4156171 & $20: 34: 28.941$ & $+41: 56: 17.09$ & O9V & 34770 & 4.86 \\
\hline J20462826+4223417 & $20: 46: 28.255$ & $+42: 23: 41.74$ & O9V & 34770 & 4.88 \\
\hline J20272553+3929246 & $20: 27: 25.529$ & $+39: 29: 24.58$ & $09.5 \mathrm{~V}$ & 33960 & 4.88 \\
\hline $\mathrm{J} 20325571+4307583$ & $20: 32: 55.713$ & $+43: 07: 58.26$ & $09.5 \mathrm{~V}$ & 33960 & 4.97 \\
\hline $\mathrm{J} 20310838+4202422$ & 20:31:08.376 & $+42: 02: 42.25$ & O9.7II & 30859 & 5.15 \\
\hline HD 199021 & $20: 52: 53.207$ & $+42: 36: 27.87$ & B0V & 32816 & 5.05 \\
\hline $\mathrm{J} 20382040+4156563$ & 20:38:20.413 & $+41: 56: 56.51$ & B0II & 29054 & 5.29 \\
\hline $\mathrm{J} 20385918+4202395$ & $20: 38: 59.181$ & $+42: 02: 39.45$ & BOIb & 27800 & 4.67 \\
\hline J20294195+3859342 & 20:29:41.952 & $+38: 59: 34.16$ & $\mathrm{~B} 0.2 \mathrm{~V}$ & 31906 & 4.61 \\
\hline $\mathrm{J} 20352227+4355305$ & $20: 35: 22.266$ & $+43: 55: 30.46$ & B0.2IV & 30588 & 4.90 \\
\hline $\mathrm{BD}+413794$ & 20:32:02.204 & $+42: 12: 26.15$ & B0.2III & 29270 & 4.97 \\
\hline HD 194839 & $20: 26: 21.545$ & $+41: 22: 45.65$ & B0.5Iae & 25014 & 5.73 \\
\hline $\mathrm{J} 20313693+4201218$ & $20: 31: 36.921$ & $+42: 01: 21.79$ & B0.7Ib & 24014 & 4.96 \\
\hline J20301273+3904216 & $20: 30: 12.732$ & $+39: 04: 21.59$ & B1V & 27173 & 4.24 \\
\hline LSIII+4217 & $20: 35: 10.623$ & $+42: 20: 22.83$ & B1III & 24903 & 4.69 \\
\hline J20374323+4232334 & $20: 37: 43.232$ & $+42: 32: 33.40$ & B1III & 24903 & 4.13 \\
\hline $\mathrm{J} 20314215+4225532$ & $20: 31: 42.151$ & $+42: 25: 53.26$ & $\mathrm{~B} 1 \mathrm{Ib}$ & 22632 & 5.67 \\
\hline $\mathrm{BD}+373976$ & $20: 33: 49.752$ & $+38: 17: 00.06$ & $\mathrm{~B} 1.5 \mathrm{Vn}$ & 24132 & 4.17 \\
\hline $\mathrm{J} 20313853+4152585$ & $20: 31: 38.532$ & $+41: 52: 58.46$ & $\mathrm{~B} 1.5 \mathrm{~V}$ & 24132 & 4.07 \\
\hline $\mathrm{J} 20312725+4304227$ & $20: 31: 27.253$ & $+43: 04: 22.67$ & $\mathrm{~B} 1.5 \mathrm{~V}$ & 24132 & 4.33 \\
\hline BD+394189 & $20: 26: 20.922$ & $+39: 40: 10.06$ & B2p?e? & 21092 & 4.85 \\
\hline $\mathrm{BD}+413762$ & $20: 28: 15.212$ & $+42: 25: 39.14$ & $\mathrm{~B} 2 \mathrm{~V}$ & 21092 & 4.32 \\
\hline $\mathrm{J} 20452110+4223514$ & $20: 45: 21.103$ & $+42: 23: 51.37$ & $\mathrm{~B} 2 \mathrm{~V}$ & 21092 & 3.99 \\
\hline $\mathrm{J} 20264025+4233221$ & $20: 26: 40.251$ & $+42: 33: 22.09$ & B2II & 19482 & 3.67 \\
\hline HD 196489 & $20: 36: 24.259$ & $+39: 11: 40.70$ & $\mathrm{~B} 3 \mathrm{~V}$ & 18546 & 3.92 \\
\hline $\mathrm{J} 20462289+4212311$ & 20:46:22.892 & $+42: 12: 31.07$ & B3 V & 18546 & 4.06 \\
\hline HD 194779 & $20: 25: 55.077$ & $+41: 20: 11.73$ & B3II & 16983 & 4.12 \\
\hline $\mathrm{BD}+384098$ & $20: 27: 33.010$ & $+38: 46: 19.62$ & $\mathrm{~B} 9 \mathrm{Ib}$ & 11457 & 3.92 \\
\hline
\end{tabular}


S. R. Berlanas et al.: New massive members of Cygnus OB2

Appendix B: Candidate spectra

In Fig. B.1 are plotted the normalized spectra of the 61 OB can- didates from the list of Comerón \& Pasquali (2012). The spectra have been corrected for the stellar radial velocities and diagnostic lines are also indicated.

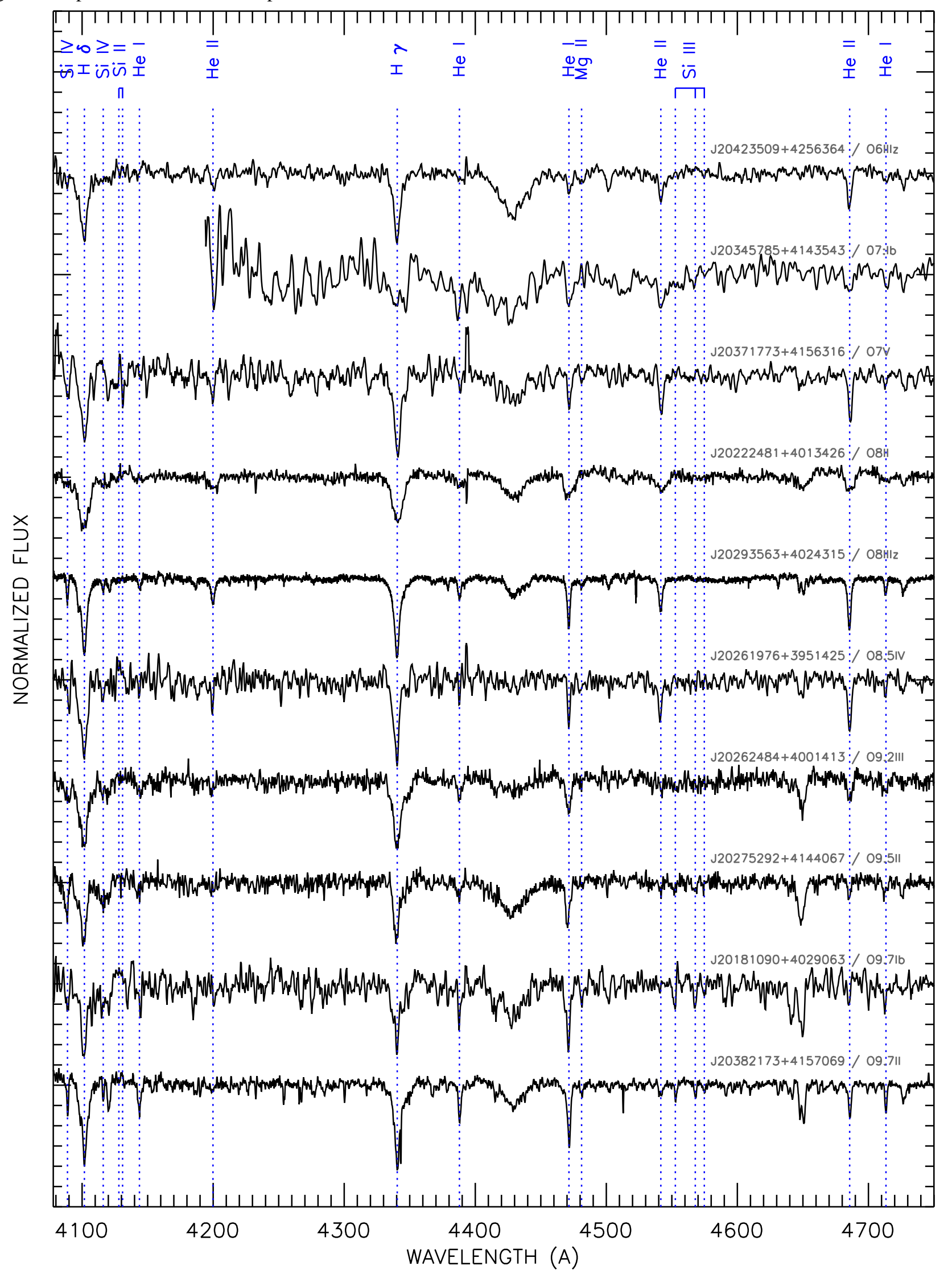

Fig. B.1. Spectra of the $61 \mathrm{OB}$ candidate stars where dotted vertical lines indicate $\mathrm{H}, \mathrm{He}, \mathrm{Si}$ and $\mathrm{Mg}$ lines in the wavelength range. 


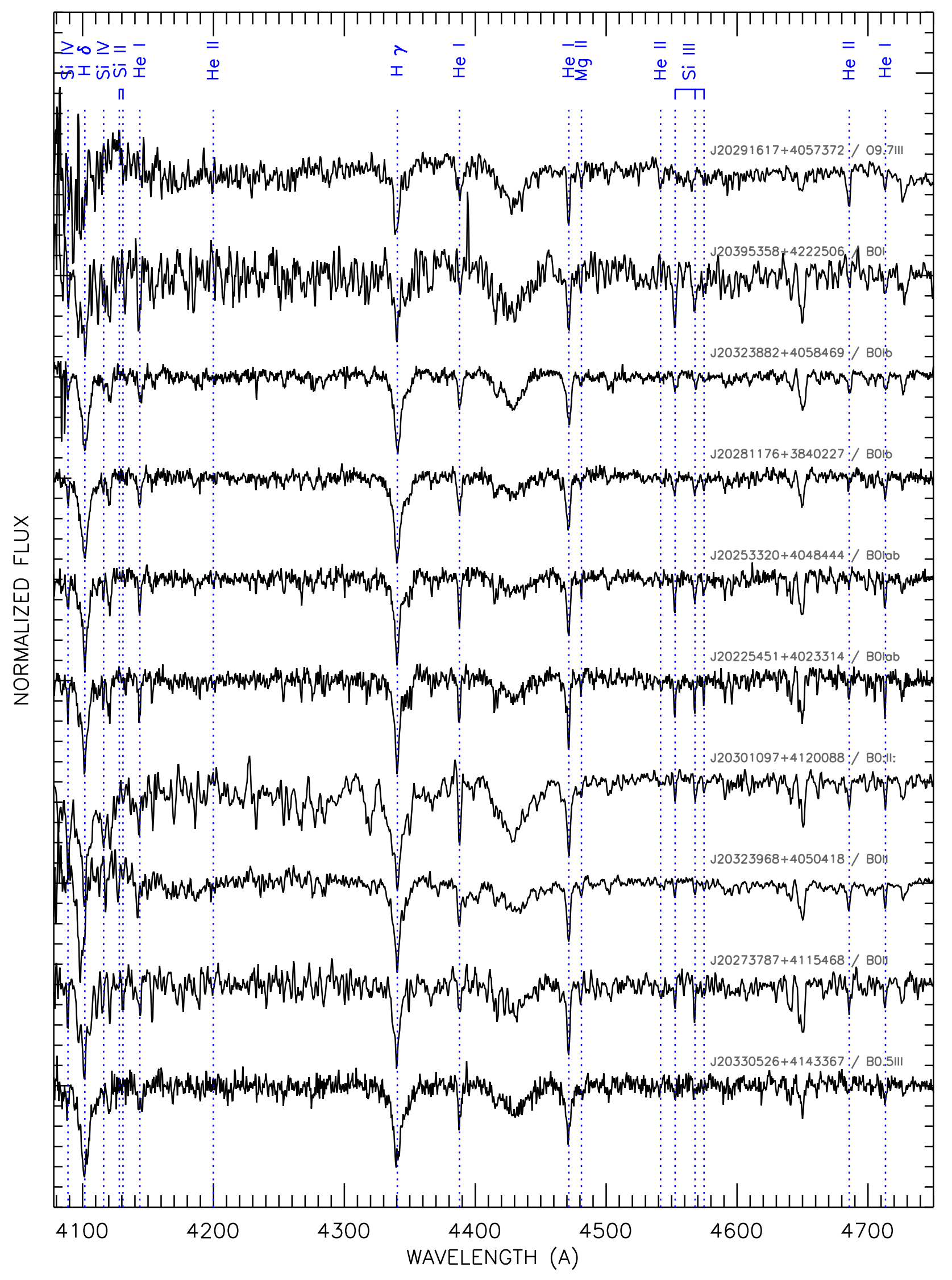

Fig. B.1. continued. 
S. R. Berlanas et al.: New massive members of Cygnus OB2

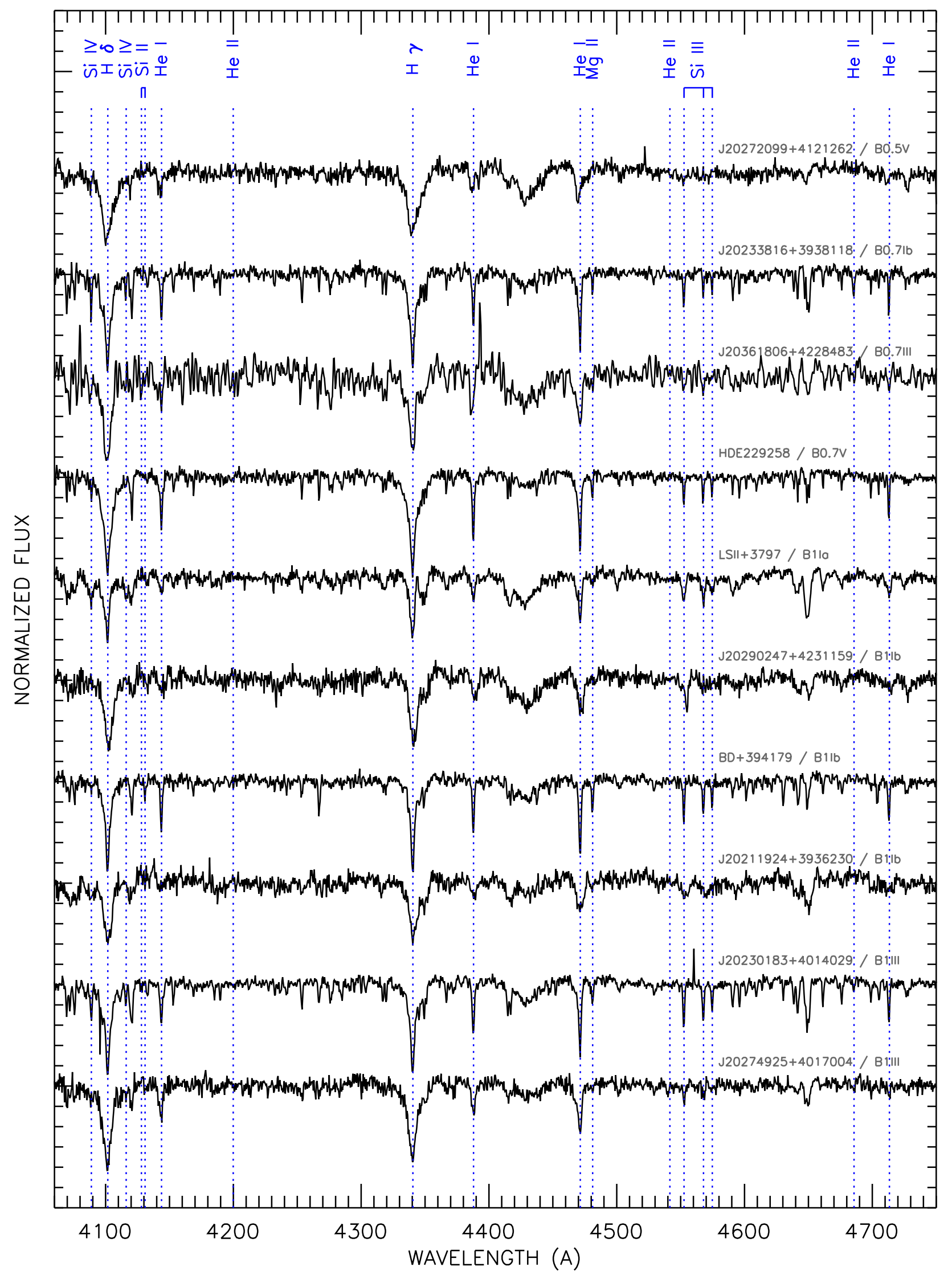

Fig. B.1. continued. 


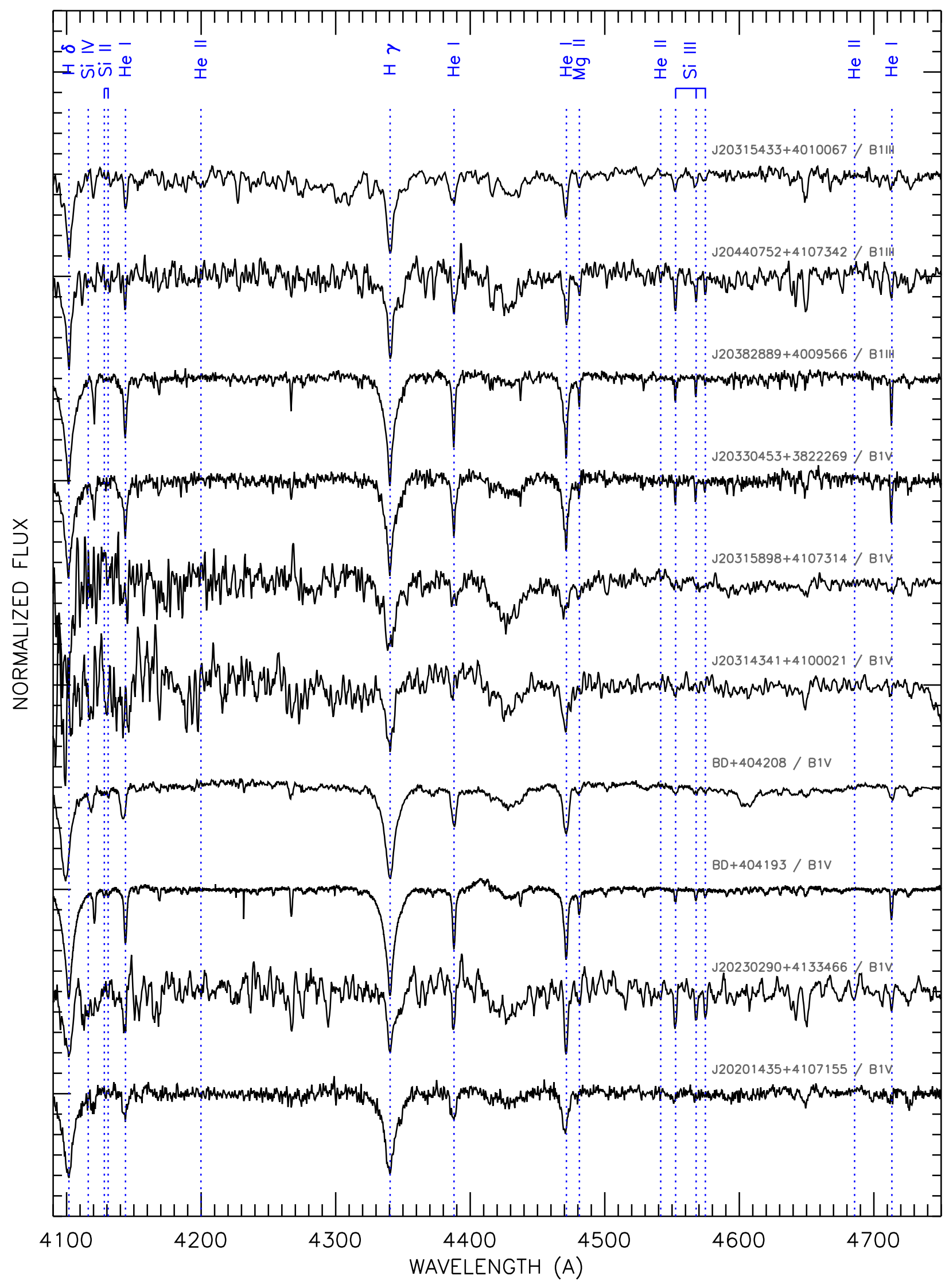

Fig. B.1. continued. 
S. R. Berlanas et al.: New massive members of Cygnus OB2

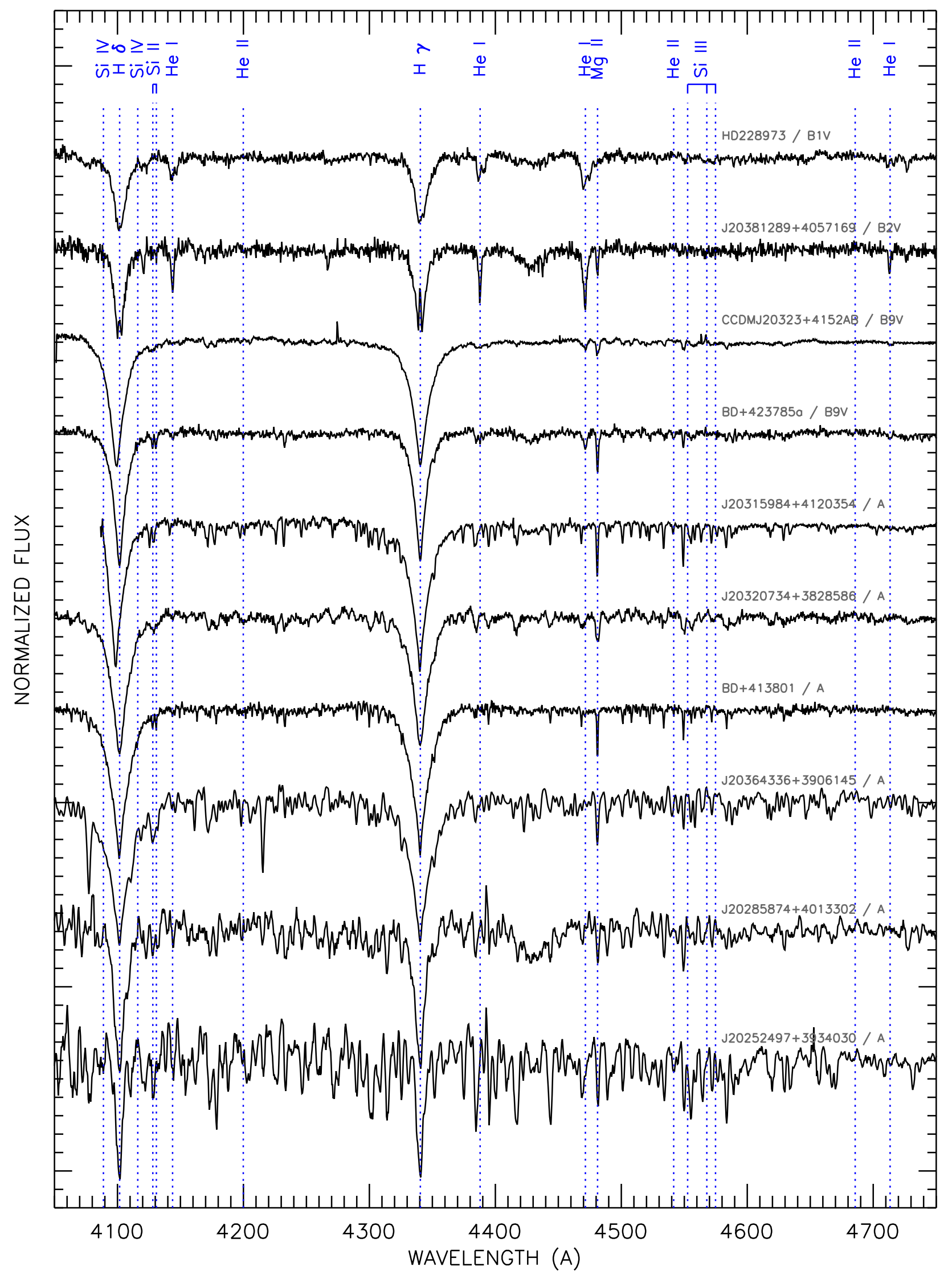

Fig. B.1. continued. 


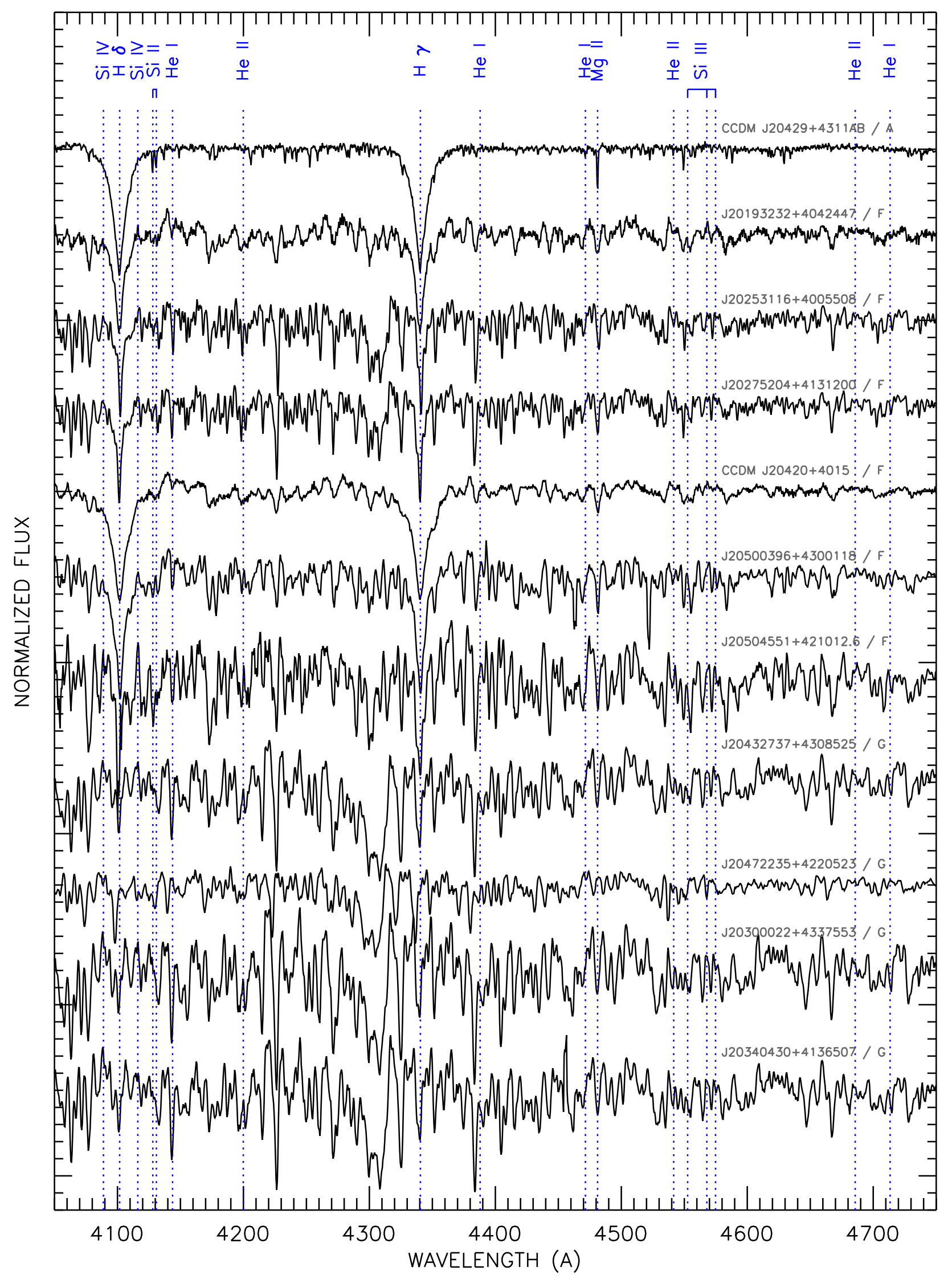

Fig. B.1. continued. 